\title{
Evolutionary Predictions Should be Based on Individual-Level Traits
}

Claus Rueffler (rueffler@zoo.utoronto.ca)

Martijn Egas (egas@science.uva.nl)

Johan A.J. Metz (j.a.j.metz@biology.leidenuniv.nl)

Approved by

Ulf Dieckmann

Program Leader, Evolution and Ecology Program

December 2006 


\section{IIASA STUDIES IN ADAPTIVE DYNAMICS No. 127}

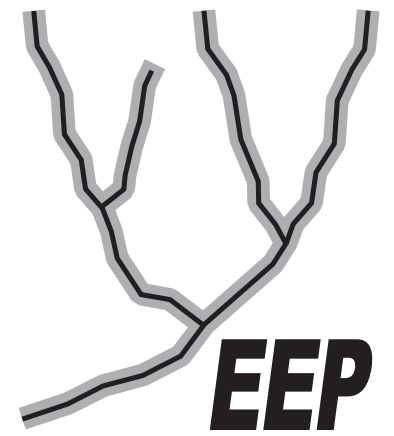

The Evolution and Ecology Program at IIASA fosters the development of new mathematical and conceptual techniques for understanding the evolution of complex adaptive systems.

Focusing on these long-term implications of adaptive processes in systems of limited growth, the Evolution and Ecology Program brings together scientists and institutions from around the world with IIASA acting as the central node.

Scientific progress within the network is collected in the IIASA Studies in Adaptive Dynamics series.
No. 1 Metz JAJ, Geritz SAH, Meszéna G, Jacobs FJA, van Heerwaarden JS: Adaptive Dynamics: A Geometrical Study of the Consequences of Nearly Faithful Reproduction. IIASA Working Paper WP-95-099 (1995). van Strien SJ, Verduyn Lunel SM (eds): Stochastic and Spatial Structures of Dynamical Systems, Proceedings of the Royal Dutch Academy of Science (KNAW Verhandelingen), North Holland, Amsterdam, pp. 183-231 (1996).

No. 2 Dieckmann U, Law R: The Dynamical Theory of Coevolution: A Derivation from Stochastic Ecological Processes. IIASA Working Paper WP-96-001 (1996). Journal of Mathematical Biology 34:579-612 (1996).

No. 3 Dieckmann U, Marrow P, Law R: Evolutionary Cycling of Predator-PreyInteractions: Population Dynamics and the Red Queen. IIASA Preprint (1995). Journal of Theoretical Biology 176:91-102 (1995).

No. 4 Marrow P, Dieckmann U, Law R: Evolutionary Dynamics of Predator-Prey Systems: An Ecological Perspective. IIASA Working Paper WP-96-002 (1996). Journal of Mathematical Biology 34:556-578 (1996).

No. 5 Law R, Marrow P, Dieckmann U: On Evolution under Asymmetric Competition. IIASA Working Paper WP-96-003 (1996). Evolutionary Ecology 11:485-501 (1997).

No. 6 Metz JAJ, Mylius SD, Diekmann O: When Does Evolution Optimize? On the Relation Between Types of Density Dependence and Evolutionarily Stable Life History Parameters. IIASA Working Paper WP-96-004 (1996).

No. 7 Ferrière R, Gatto M: Lyapunov Exponents and the Mathematics of Invasion in Oscillatory or Chaotic Populations. Theoretical Population Biology 48:126-171 (1995).

No. 8 Ferrière R, Fox GA: Chaos and Evolution. IIASA Preprint (1996). Trends in Ecology and Evolution 10:480485 (1995).

No. 9 Ferrière R, Michod RE: The Evolution of Cooperation in Spatially Heterogeneous Populations. IIASA Working Paper WP-96-029 (1996). The American Naturalist 147:692717 (1996).

No. 10 van Dooren TJM, Metz JAJ: Delayed Maturation in Temporally Structured Populations with Non-Equilibrium Dynamics. IIASA Working Paper WP-96-070 (1996). Journal of Evolutionary Biology 11:41-62 (1998).
No. 11 Geritz SAH, Metz JAJ, Kisdi É, Meszéna G: The Dynamics of Adaptation and Evolutionary Branching. IIASA Working Paper WP-96-077 (1996). Physical Review Letters 78:2024-2027 (1997).

No. 12 Geritz SAH, Kisdi É, Meszéna G, Metz JAJ: Evolutionary Singular Strategies and the Adaptive Growth and Branching of the Evolutionary Tree. IIASA Working Paper WP-96-114 (1996). Evolutionary Ecology 12:35-57 (1998).

No. 13 Heino M, Metz JAJ, Kaitala V: Evolution of Mixed Maturation Strategies in Semelparous Life-Histories: The Crucial Role of Dimensionality of Feedback Environment. IIASA Working Paper WP-96-126 (1996). Philosophical Transactions of the Royal Society of London Series B 352:1647-1655 (1997).

No. 14 Dieckmann U: Can Adaptive Dynamics Invade? IIASA Working Paper WP-96-152 (1996). Trends in Ecology and Evolution 12:128-131 (1997).

No. 15 Meszéna G, Czibula I, Geritz SAH: Adaptive Dynamics in a 2-Patch Environment: A Simple Model for Allopatric and Parapatric Speciation. IIASA Interim Report IR-97-001 (1997). Journal of Biological Systems 5:265-284 (1997).

No. 16 Heino M, Metz JAJ, Kaitala V: The Enigma of Frequency-Dependent Selection. IIASA Interim Report IR97-061 (1997). Trends in Ecology and Evolution 13:367-370 (1998).

No. 17 Heino M: Management of Evolving Fish Stocks. IIASA Interim Report IR-97-062 (1997). Canadian Journal of Fisheries and Aquatic Sciences 55:1971-1982 (1998).

No. 18 Heino M: Evolution of Mixed Reproductive Strategies in Simple Life-History Models. IIASA Interim Report IR-97063 (1997).

No. 19 Geritz SAH, van der Meijden E, Metz JAJ: Evolutionary Dynamics of Seed Size and Seedling Competitive Ability. IIASA Interim Report IR-97-071 (1997). Theoretical Population Biology 55:324-343 (1999).

No. 20 Galis F, Metz JAJ: Why Are There So Many Cichlid Species? On the Interplay of Speciation and Adaptive Radiation. IIASA Interim Report IR-97-072 (1997). Trends in Ecology and Evolution 13:1-2 (1998). 
No. 21 Boerlijst MC, Nowak MA, Sigmund K: Equal Pay for all Prisoners/ The Logic of Contrition. IIASA Interim Report IR-97-073 (1997). American Mathematical Society Monthly 104:303-307 (1997). Journal of Theoretical Biology 185:281-293 (1997).

No. 22 Law R, Dieckmann U: Symbiosis Without Mutualism and the Merger of Lineages in Evolution. IIASA Interim Report IR-97-074 (1997). Proceedings of the Royal Society of London Series B 265:1245-1253 (1998).

No. 23 Klinkhamer PGL, de Jong TJ, Metz JAJ: Sex and Size in Cosexual Plants. IIASA Interim Report IR-97-078 (1997). Trends in Ecology and Evolution 12:260-265 (1997).

No. 24 Fontana W, Schuster P: Shaping Space: The Possible and the Attainable in RNA Genotype-Phenotype Mapping. IIASA Interim Report IR-98-004 (1998). Journal of Theoretical Biology 194:491-515 (1998).

No. 25 Kisdi É, Geritz SAH: Adaptive Dynamics in Allele Space: Evolution of Genetic Polymorphism by Small Mutations in a Heterogeneous Environment. IIASA Interim Report IR-98-038 (1998). Evolution 53:993-1008 (1999).

No. 26 Fontana W, Schuster P: Continuity in Evolution: On the Nature of Transitions. IIASA Interim Report IR-98-039 (1998). Science 280:1451-1455 (1998).

No. 27 Nowak MA, Sigmund K: Evolution of Indirect Reciprocity by Image Scoring/ The Dynamics of Indirect Reciprocity. IIASA Interim Report IR-98-040 (1998). Nature 393:573-577 (1998). Journal of Theoretical Biology 194:561574 (1998).

No. 28 Kisdi É: Evolutionary Branching Under Asymmetric Competition. IIASA Interim Report IR-98-045 (1998). Journal of Theoretical Biology 197:149-162 (1999).

No. 29 Berger U: Best Response Adaptation for Role Games. IIASA Interim Report IR-98-086 (1998).

No. 30 van Dooren TJM: The Evolutionary Ecology of Dominance-Recessivity. IIASA Interim Report IR-98-096 (1998). Journal of Theoretical Biology 198:519-532 (1999).

No. 31 Dieckmann U, O'Hara B, Weisser W: The Evolutionary Ecology of Dispersal. IIASA Interim Report IR-98-108 (1998). Trends in Ecology and Evolution 14:88-90 (1999).

No. 32 Sigmund K: Complex Adaptive Systems and the Evolution of Reciprocation. IIASA Interim Report IR-98-100 (1998). Ecosystems 1:444-448 (1998).

No. 33 Posch M, Pichler A, Sigmund K: The Efficiency of Adapting Aspiration Levels. IIASA Interim Report IR-98103 (1998). Proceedings of the Royal Society London Series B 266:1427-1435 (1999).

No. 34 Mathias A, Kisdi É: Evolutionary Branching and Coexistence of Germination Strategies. IIASA Interim Report IR-99-014 (1999).

No. 35 Dieckmann U, Doebeli M: On the Origin of Species by Sympatric Speciation. IIASA Interim Report IR-99-013 (1999). Nature 400:354-357 (1999).

No. 36 Metz JAJ, Gyllenberg M: How Should We Define Fitness in Structured Metapopulation Models? Including an Application to the Calculation of Evolutionarily Stable Dispersal Strategies. IIASA Interim Report IR-99-019 (1999). Proceedings of the Royal Society of London Series B 268:499508 (2001)
No. 37 Gyllenberg M, Metz JAJ: On Fitness in Structured Metapopulations. IIASA Interim Report IR-99-037 (1999). Journal of Mathematical Biology 43:545-560 (2001).

No. 38 Meszéna G, Metz JAJ: Species Diversity and Population Regulation: The Importance of Environmental Feedback Dimensionality. IIASA Interim Report IR-99-045 (1999).

No. 39 Kisdi É, Geritz SAH: Evolutionary Branching and Sympatric Speciation in Diploid Populations. IIASA Interim Report IR-99-048 (1999).

No. 40 Ylikarjula J, Heino M, Dieckmann U: Ecology and Adaptation of Stunted Growth in Fish. IIASA Interim Report IR-99-050 (1999). Evolutionary Ecology 13:433-453 (1999).

No. 41 Nowak MA, Sigmund K: Games on Grids. IIASA Interim Report IR-99-038 (1999). Dieckmann U, Law R, Metz JAJ (eds): The Geometry of Ecological Interactions: Simplifying Spatial Complexity, Cambridge University Press, Cambridge, UK, pp. 135-150 (2000).

No. 42 Ferrière R, Michod RE: Wave Patterns in Spatial Games and the Evolution of Cooperation. IIASA Interim Report IR-99-041 (1999). Dieckmann U, Law R, Metz JAJ (eds): The Geometry of Ecological Interactions: Simplifying Spatial Complexity, Cambridge University Press, Cambridge, UK, pp. 318-332 (2000).

No. 43 Kisdi É, Jacobs FJA, Geritz SAH: Red Queen Evolution by Cycles of Evolutionary Branching and Extinction. IIASA Interim Report IR-00-030 (2000). Selection 2:161$176(2001)$.

No. 44 Meszéna G, Kisdi É, Dieckmann U, Geritz SAH, Metz JAJ: Evolutionary Optimisation Models and Matrix Games in the Unified Perspective of Adaptive Dynamics. IIASA Interim Report IR-00-039 (2000). Selection 2:193-210 (2001).

No. 45 Parvinen K, Dieckmann U, Gyllenberg M, Metz JAJ: Evolution of Dispersal in Metapopulations with Local Density Dependence and Demographic Stochasticity. IIASA Interim Report IR-00-035 (2000). Journal of Evolutionary Biology 16:143-153 (2003).

No. 46 Doebeli M, Dieckmann U: Evolutionary Branching and Sympatric Speciation Caused by Different Types of Ecological Interactions. IIASA Interim Report IR-00-040 (2000). The American Naturalist 156:S77-S101 (2000).

No. 47 Heino M, Hanski I: Evolution of Migration Rate in a Spatially Realistic Metapopulation Model. IIASA Interim Report IR-00-044 (2000). The American Naturalist 157:495$511(2001)$.

No. 48 Gyllenberg M, Parvinen K, Dieckmann U: Evolutionary Suicide and Evolution of Dispersal in Structured Metapopulations. IIASA Interim Report IR-00-056 (2000). Journal of Mathematical Biology 45:79-105 (2002).

No. 49 van Dooren TJM: The Evolutionary Dynamics of Direct Phenotypic Overdominance: Emergence Possible, Loss Probable. IIASA Interim Report IR-00-048 (2000). Evolution 54:1899-1914 (2000).

No. 50 Nowak MA, Page KM, Sigmund K: Fairness Versus Reason in the Ultimatum Game. IIASA Interim Report IR00-57 (2000). Science 289:1773-1775 (2000).

No. 51 de Feo O, Ferrière R: Bifurcation Analysis of Population Invasion: On-Off Intermittency and Basin Riddling. IIASA Interim Report IR-00-074 (2000). International Journal of Bifurcation and Chaos 10:443-452 (2000). 
No. 52 Heino M, Laaka-Lindberg S: Clonal Dynamics and Evolution of Dormancy in the Leafy Hepatic Lophozia Silvicola. IIASA Interim Report IR-01-018 (2001). Oikos 94:525-532 (2001).

No. 53 Sigmund K, Hauert C, Nowak MA: Reward and Punishment in Minigames. IIASA Interim Report IR-01-031 (2001). Proceedings of the National Academy of Sciences of the USA 98:10757-10762 (2001).

No. 54 Hauert C, De Monte S, Sigmund K, Hofbauer J: Oscillations in Optional Public Good Games. IIASA Interim Report IR-01-036 (2001).

No. 55 Ferrière R, Le Galliard J: Invasion Fitness and Adaptive Dynamics in Spatial Population Models. IIASA Interim Report IR-01-043 (2001). Clobert J, Dhondt A, Danchin E, Nichols J (eds): Dispersal, Oxford University Press, pp. 57-79 (2001).

No. 56 de Mazancourt C, Loreau M, Dieckmann U: Can the Evolution of Plant Defense Lead to Plant-Herbivore Mutualism? IIASA Interim Report IR-01-053 (2001). The American Naturalist 158:109-123 (2001).

No. 57 Claessen D, Dieckmann U: Ontogenetic Niche Shifts and Evolutionary Branching in Size-Structured Populations. IIASA Interim Report IR-01-056 (2001). Evolutionary Ecology Research 4:189-217 (2002).

No. 58 Brandt H: Correlation Analysis of Fitness Landscapes. IIASA Interim Report IR-01-058 (2001).

No. 59 Dieckmann U: Adaptive Dynamics of Pathogen-Host Interacations. IIASA Interim Report IR-02-007 (2002). Dieckmann U, Metz JAJ, Sabelis MW, Sigmund K (eds): Adaptive Dynamics of Infectious Diseases: In Pursuit of Virulence Management, Cambridge University Press, Cambridge, UK, pp. 39-59 (2002).

No. 60 Nowak MA, Sigmund K: Super- and Coinfection: The Two Extremes. IIASA Interim Report IR-02-008 (2002). Dieckmann U, Metz JAJ, Sabelis MW, Sigmund K (eds): Adaptive Dynamics of Infectious Diseases: In Pursuit of Virulence Management, Cambridge University Press, Cambridge, UK, pp. 124-137 (2002).

No. 61 Sabelis MW, Metz JAJ: Evolution Management: Taking Stock - Relating Theory to Experiment. IIASA Interim Report IR-02-009 (2002). Dieckmann U, Metz JAJ, Sabelis MW, Sigmund K (eds): Adaptive Dynamics of Infectious Diseases: In Pursuit of Virulence Management, Cambridge University Press, Cambridge, UK, pp. 379-398 (2002).

No. 62 Cheptou P, Dieckmann U: The Evolution of SelfFertilization in Density-Regulated Populations . IIASA Interim Report IR-02-024 (2002). Proceedings of the Royal Society of London Series B 269:1177-1186(2002).

No. 63 Bürger R: Additive Genetic Variation Under Intraspecific Competition and Stabilizing Selection: A Two-Locus Study. IIASA Interim Report IR-02-013 (2002). Theoretical Population Biology 61:197-213 (2002).

No. 64 Hauert C, De Monte S, Hofbauer J, Sigmund K: Volunteering as Red Queen Mechanism for Co-operation in Public Goods Games. IIASA Interim Report IR-02-041 (2002). Science 296:1129-1132 (2002).

No. 65 Dercole F, Ferrière R, Rinaldi S: Ecological Bistability and Evolutionary Reversals under Asymmetrical Competition. IIASA Interim Report IR-02-053 (2002). Evolution 56:1081-1090 (2002).
No. 66 Dercole F, Rinaldi S: Evolution of Cannibalistic Traits: Scenarios Derived from Adaptive Dynamics. IIASA Interim Report IR-02-054 (2002). Theoretical Population Biology 62:365-374 (2002).

No. 67 Bürger R, Gimelfarb A: Fluctuating Environments and the Role of Mutation in Maintaining Quantitative Genetic Variation. IIASA Interim Report IR-02-058 (2002). Genetical Research 80:31-46 (2002).

No. 68 Bürger R: On a Genetic Model of Intraspecific Competition and Stabilizing Selection. IIASA Interim Report IR02-062 (2002). Amer. Natur. 160:661-682 (2002).

No. 69 Doebeli M, Dieckmann U: Speciation Along Environmental Gradients. IIASA Interim Report IR-02-079 (2002). Nature 421:259-264 (2003).

No. 70 Dercole F, Irisson J, Rinaldi S: Bifurcation Analysis of a Prey-Predator Coevolution Model. IIASA Interim Report IR-02-078 (2002). SIAM Journal on Applied Mathematics 63:1378-1391 (2003).

No. 71 Le Galliard J, Ferrière R, Dieckmann U: The Adaptive Dynamics of Altruism in Spatially Heterogeneous Populations. IIASA Interim Report IR-03-006 (2003). Evolution 57:1-17 (2003).

No. 72 Taborsky B, Dieckmann U, Heino M: Unexpected Discontinuities in Life-History Evolution under SizeDependent Mortality. IIASA Interim Report IR-03-004 (2003). Proceedings of the Royal Society of London Series B 270:713-721 (2003).

No. 73 Gardmark A, Dieckmann U, Lundberg P: LifeHistory Evolution in Harvested Populations: The Role of Natural Predation. IIASA Interim Report IR-03-008 (2003). Evolutionary Ecology Research 5:239-257 (2003).

No. 74 Mizera F, Meszéna G: Spatial Niche Packing, Character Displacement and Adaptive Speciation Along an Environmental Gradient. IIASA Interim Report IR-03-062 (2003). Evolutionary Ecology Research 5:363-382 (2003).

No. 75 Dercole F: Remarks on Branching-Extinction Evolutionary Cycles. IIASA Interim Report IR-03-077 (2003). Journal of Mathematical Biology 47:569-580 (2003).

No. 76 Hofbauer J, Sigmund K: Evolutionary Game Dynamics. IIASA Interim Report IR-03-078 (2003). Bulletin of the American Mathematical Society 40:479-519 (2003).

No. 77 Ernande B, Dieckmann U, Heino M: Adaptive Changes in Harvested Populations: Plasticity and Evolution of Age and Size at Maturation. IIASA Interim Report IR03-058 (2003). Proceedings of the Royal Society of London Series B-Biological Sciences 271:415-423 (2004).

No. 78 Hanski I, Heino M: Metapopulation-Level Adaptation of Insect Host Plant Preference and Extinction-Colonization Dynamics in Heterogeneous Landscapes. IIASA Interim Report IR-03-028 (2003). Theoretical Population Biology 63:309-338 (2003).

No. 79 van Doorn G, Dieckmann U, Weissing FJ: Sympatric Speciation by Sexual Selection: A Critical Re-Evaluation. IIASA Interim Report IR-04-003 (2004). American Naturalist 163:709-725 (2004).

No. 80 Egas M, Dieckmann U, Sabelis MW: Evolution Restricts the Coexistence of Specialists and Generalists - the Role of Trade-off Structure. IIASA Interim Report IR-04-004 (2004). American Naturalist 163:518-531 (2004). 
No. 81 Ernande B, Dieckmann U: The Evolution of Phenotypic Plasticity in Spatially Structured Environments: Implications of Intraspecific Competition, Plasticity Costs, and Environmental Characteristics. IIASA Interim Report IR-04-006 (2004). Journal of Evolutionary Biology 17:613-628 (2004).

No. 82 Cressman R, Hofbauer J: Measure Dynamics on a One-Dimensional Continuous Trait Space: Theoretical Foundations for Adaptive Dynamics. IIASA Interim Report IR04-016 (2004).

No. 83 Cressman R: Dynamic Stability of the Replicator Equation with Continuous Strategy Space. IIASA Interim Report IR-04-017 (2004).

No. 84 Ravigné V, Olivieri I, Dieckmann U: Implications of Habitat Choice for Protected Polymorphisms. IIASA Interim Report IR-04-005 (2004). Evolutionary Ecology Research 6:125-145 (2004).

No. 85 Nowak MA, Sigmund K: Evolutionary Dynamics of Biological Games. IIASA Interim Report IR-04-013 (2004). Science 303:793-799 (2004).

No. 86 Vukics A, Asbóth J, Meszéna G: Speciation in Multidimensional Evolutionary Space. IIASA Interim Report IR-04-028 (2004). Physical Review 68:041-903 (2003).

No. 87 de Mazancourt C, Dieckmann U: Trade-off Geometries and Frequency-dependent Selection. IIASA Interim Report IR-04-039 (2004). American Naturalist 164:765-778 (2004).

No. 88 Cadet CR, Metz JAJ, Klinkhamer PGL: Size and the Not-So-Single Sex: Disentangling the Effects of Size on Sex Allocation. IIASA Interim Report IR-04-084 (2004). American Naturalist 164:779-792 (2004).

No. 89 Rueffler C, van Dooren TJM, Metz JAJ: Adaptive Walks on Changing Landscapes: Levins' Approach Extended. IIASA Interim Report IR-04-083 (2004). Theoretical Population Biology 65:165-178 (2004).

No. 90 de Mazancourt C, Loreau M, Dieckmann U: Understanding Mutualism When There is Adaptation to the Partner. IIASA Interim Report IR-05-016 (2005). Journal of Ecology 93:305-314 (2005).

No. 91 Dieckmann U, Doebeli M: Pluralism in Evolutionary Theory. IIASA Interim Report IR-05-017 (2005). Journal of Evolutionary Biology 18:1209-1213 (2005).

No. 92 Doebeli M, Dieckmann U, Metz JAJ, Tautz D: What We Have Also Learned: Adaptive Speciation is Theoretically Plausible. IIASA Interim Report IR-05-018 (2005). Evolution 59:691-695 (2005).

No. 93 Egas M, Sabelis MW, Dieckmann U: Evolution of Specialization and Ecological Character Displacement of Herbivores Along a Gradient of Plant Quality. IIASA Interim Report IR-05-019 (2005). Evolution 59:507-520 (2005).

No. 94 Le Galliard J, Ferrière R, Dieckmann U: Adaptive Evolution of Social Traits: Origin, Trajectories, and Correlations of Altruism and Mobility. IIASA Interim Report IR05-020 (2005). American Naturalist 165:206-224 (2005).

No. 95 Doebeli M, Dieckmann U: Adaptive Dynamics as a Mathematical Tool for Studying the Ecology of Speciation Processes. IIASA Interim Report IR-05-022 (2005). Journal of Evolutionary Biology 18:1194-1200 (2005).

No. 96 Brandt H, Sigmund K: The Logic of Reprobation: Assessment and Action Rules for Indirect Reciprocity. IIASA Interim Report IR-04-085 (2004). Journal of Theoretical Biology 231:475-486 (2004).
No. 97 Hauert C, Haiden N, Sigmund K: The Dynamics of Public Goods. IIASA Interim Report IR-04-086 (2004). Discrete and Continuous Dynamical Systems - Series B 4:575587 (2004).

No. 98 Meszéna G, Gyllenberg M, Jacobs FJA, Metz JAJ: Link Between Population Dynamics and Dynamics of Darwinian Evolution. IIASA Interim Report IR-05-026 (2005). Physical Review Letters 95:Article 078105 (2005).

No. 99 Meszéna G: Adaptive Dynamics: The Continuity Argument. IIASA Interim Report IR-05-032 (2005).

No. 100 Brännström NA, Dieckmann U: Evolutionary Dynamics of Altruism and Cheating Among Social Amoebas. IIASA Interim Report IR-05-039 (2005). Proceedings of the Royal Society London Series B 272:1609-1616 (2005).

No. 101 Meszéna G, Gyllenberg M, Pasztor L, Metz JAJ: Competitive Exclusion and Limiting Similarity: A Unified Theory. IIASA Interim Report IR-05-040 (2005).

No. 102 Szabo P, Meszéna G: Limiting Similarity Revisited. IIASA Interim Report IR-05-050 (2005).

No. 103 Krakauer DC, Sasaki A: The Greater than Two-Fold Cost of Integration for Retroviruses. IIASA Interim Report IR-05-069 (2005).

No. 104 Metz JAJ: Eight Personal Rules for Doing Science. IIASA Interim Report IR-05-073 (2005). Journal of Evolutionary Biology 18:1178-1181 (2005).

No. 105 Beltman JB, Metz JAJ: Speciation: More Likely Through a Genetic or Through a Learned Habitat Preference? IIASA Interim Report IR-05-072 (2005). Proceedings of the Royal Society of London Series B 272:1455-1463 (2005).

No. 106 Durinx M, Metz JAJ: Multi-type Branching Processes and Adaptive Dynamics of Structured Populations. IIASA Interim Report IR-05-074 (2005). Haccou P, Jager P, Vatutin V (eds): Branching Processes: Variation, Growth and Extinction of Populations, Cambridge University Press, Cambridge, UK, pp. 266-278 (2005).

No. 107 Brandt H, Sigmund K: The Good, the Bad and the Discriminator - Errors in Direct and Indirect Reciprocity. IIASA Interim Report IR-05-070 (2005). Journal of Theoretical Biology 239:183-194 (2006).

No. 108 Brandt H, Hauert C, Sigmund K: Punishing and Abstaining for Public Goods. IIASA Interim Report IR-05-071 (2005). Proceedings of the National Academy of Sciences of the United States of America 103:495-497 (2006).

No. 109 Ohtsuki A, Sasaki A: Epidemiology and DiseaseControl Under Gene-for-Gene Plant-Pathogen Interaction. IIASA Interim Report IR-05-068 (2005).

No. 110 Brandt H, Sigmund K: Indirect Reciprocity, ImageScoring, and Moral Hazard. IIASA Interim Report IR-05078 (2005). Proceedings of the National Academy of Sciences of the United States of America 102:2666-2670 (2005).

No. 111 Nowak MA, Sigmund K: Evolution of Indirect Reciprocity. IIASA Interim Report IR-05-079 (2005). Nature 437:1292-1298 (2005).

No. 112 Kamo M, Sasaki A: Evolution Towards Multi-Year Periodicity in Epidemics. IIASA Interim Report IR-05-080 (2005). Ecology Letters 8:378-385 (2005). 
No. 113 Dercole F, Ferrière R, Gragnani A, Rinaldi S: Coevolution of Slow-fast Populations: Evolutionary Sliding, Evolutionoary Pseudo-equilibria, and Complex Red Queen Dynamics. IIASA Interim Report IR-06-006 (2006). Proceedings of the Royal Society B-Biological Sciences 273:983-990 (2006).

No. 114 Dercole F: Border Collision Bifurcations in the Evolution of Mutualistic Interactions. IIASA Interim Report IR-05-083 (2005). International Journal of Bifurcation and Chaos 15:2179-2190 (2005).

No. 115 Dieckmann U, Heino M, Parvinen K: The Adaptive Dynamics of Function-Valued Traits. IIASA Interim Report IR-06-036 (2006). Journal of Theoretical Biology 241:370389 (2006).

No. 116 Dieckmann U, Metz JAJ: Surprising Evolutionary Predictions from Enhanced Ecological Realism. IIASA Interim Report IR-06-037 (2006). Theoretical Population Biology 69:263-281 (2006).

No. 117 Dieckmann U, Brännström NA, HilleRisLambers R, Ito $\mathrm{H}$ : The Adaptive Dynamics of Community Structure. IIASA Interim Report IR-06-038 (2006). Takeuchi Y, Iwasa Y, Sato K (eds): Mathematics for Ecology and Environmental Sciences, Springer, Berlin Heidelberg, pp. 145-177 (2007).

No. 118 Gardmark A, Dieckmann U: Disparate Maturation Adaptations to Size-dependent Mortality. IIASA Interim Report IR-06-039 (2006). Proceedings of the Royal Society London Series B 273:2185-2192 (2006).

No. 119 van Doorn G, Dieckmann U: The Long-term Evolution of Multi-locus Traits Under Frequency-dependent Disruptive Selection. IIASA Interim Report IR-06-041 (2006). Evolution 60:2226-2238 (2006).
No. 120 Doebeli M, Blok HJ, Leimar O, Dieckmann U: Multimodal Pattern Formation in Phenotype Distributions of Sexual Populations. IIASA Interim Report IR-06-046 (2006). Proceedings of the Royal Society London Series B 274:347357 (2007)

No. 121 Dunlop ES, Shuter BJ, Dieckmann U: The Demographic and Evolutionary Consequences of Selective Mortality: Predictions from an Eco-genetic Model of the Smallmouth Bass. IIASA Interim Report IR-06-060 (2006).

No. 122 Metz JAJ: Fitness. IIASA Interim Report IR-06061 (2006).

No. 123 Brandt H, Ohtsuki H, Iwasa Y, Sigmund K: A Survey on Indirect Reciprocity. IIASA Interim Report IR-06-065 (2006). Takeuchi Y, Iwasa Y, Sato K (eds): Mathematics for Ecology and Environmental Sciences, Springer, Berlin Heidelberg, pp. 21-51 (2007).

No. 124 Dercole F, Loiacono D, Rinaldi S: Synchronization in Ecological Networks: A Byproduct of Darwinian Evolution? IIASA Interim Report IR-06-068 (2006).

No. 125 Dercole F, Dieckmann U, Obersteiner M, Rinaldi S: Adaptive Dynamics and Technological Change. IIASA Interim Report IR-06-070 (2006).

No. 126 Rueffler C, van Dooren TJM, Metz JAJ: The Evolution of Resource Specialization Through FrequencyDependent and Frequency-Independent Mechanisms. IIASA Interim Report IR-06-073 (2006). American Naturalist 167:81-93 (2006).

No. 127 Rueffler C, Egas M, Metz JAJ: Evolutionary Predictions Should be Based on Individual Traits. IIASA Interim Report IR-06-074 (2006). American Naturalist 168:148-162 (2006).

Issues of the IIASA Studies in Adaptive Dynamics series can be obtained at www.iiasa.ac.at/Research/EEP/Series.html or by writing toeep@iiasa.ac.at. 


\section{Contents}

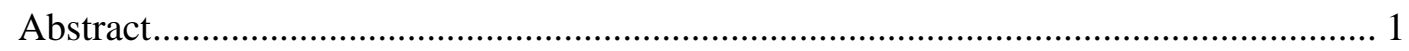

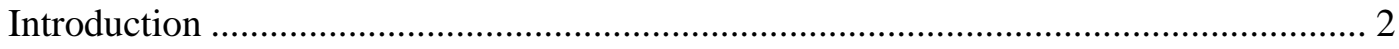

Population-Level Parameters Derive From Underlying Traits at the Level of the

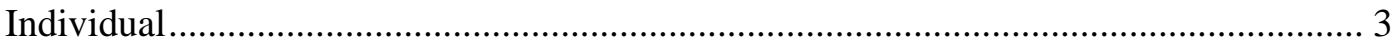

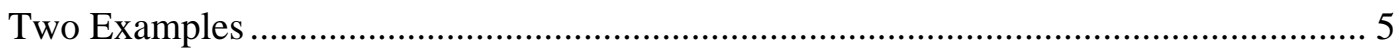

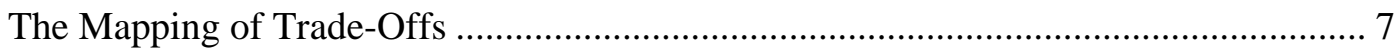

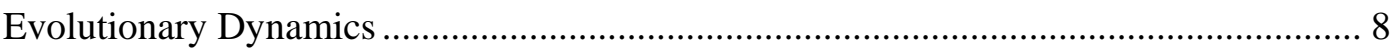

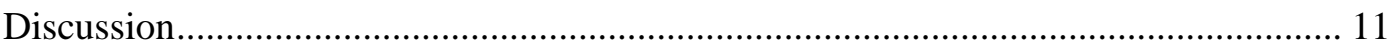

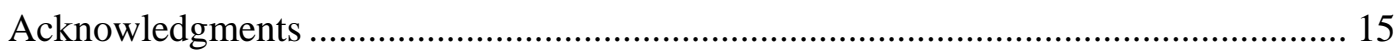

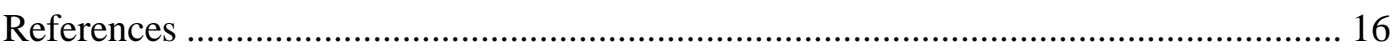

Appendix A: Fast Migration Between Habitats .......................................................... 19

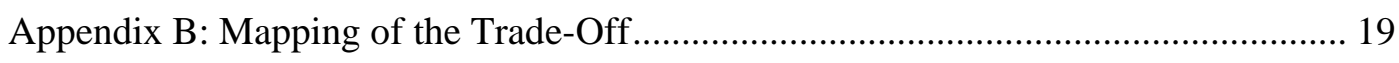

Appendix C: Analytical Results for Evolutionary Analysis...................................... 20

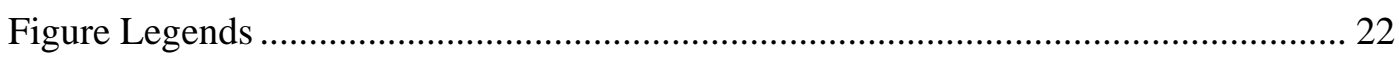


Prepared with AASTeX for the American Naturalist

\title{
Evolutionary Predictions Should be Based on Individual-Level Traits
}

\author{
Claus Rueffler ${ }^{1,2}$, Martijn Egas $^{3}$ and Johan A. J. Metz ${ }^{1,4,5}$
}

\begin{abstract}
A recent series of theoretical studies have analyzed the evolution of habitat specialization using either the logistic or the Ricker equation. These studies have implemented evolutionary change directly in population-level parameters such as habitat-specific intrinsic growth rates $r$ or carrying capacities $K$. This approach is a shortcut to a more detailed analysis where evolutionary change is studied in underlying morphological, physiological or behavioral traits at the level of the individual that contribute to $r$ or $K$. Here we describe two pitfalls that can occur when such a shortcut is employed. Firstly, population-level parameters that appear as independent variables in a population dynamical model might not be independent when derived from processes at the individual level. Secondly, patterns of covariation between individual-level traits are usually not conserved when mapped to the level of demographic parameters. Nonlinear mappings constrain the curvature of trade-offs that can sensibly be assumed at the population level. To illustrate these results we derive a two-habitat version of the logistic and the Ricker equation from individual-level processes and compare the evolutionary dynamics of habitat-specific carrying capacities with those of underlying individual-level traits contributing to the carrying capacities. Finally, we sketch how our viewpoint affects the results of earlier theoretical studies.
\end{abstract}

Subject headings: development, logistic equation, optimization, Ricker equation, specialization, trade-off

\footnotetext{
${ }^{1}$ Institute of Biology, Leiden University, Kaiserstraat 63, 2311 GP Leiden, The Netherlands

${ }^{2}$ corresponding author, present address: Department of Zoology, University of Toronto

25 Harbord St., Toronto, Ontario, M5S 3G5, Canada, rueffler@zoo.utoronto.ca

${ }^{3}$ Institute for Biodiversity and Ecosystem Dynamics, University of Amsterdam, P.O. Box 94084, 1090 GB Amsterdam, The Netherlands, egas@science.uva.nl

${ }^{4}$ 2nd affiliation: Adaptive Dynamics Network, IIASA, Laxenburg, Austria

${ }^{5}$ metz@rulsfb.leidenuniv.nl
} 


\section{Introduction}

Long term evolution by mutation and selection is largely driven by invasion of novel genotypes into resident populations. Invasion is a population dynamical process and, when evolution is studied by means of mathematical models, therefore has to be inferred from a population dynamical model. In the recent literature several studies have appeared that analyze the evolution of habitat specialization using either the Ricker equation (Wilson and Yoshimura 1994; Egas et al. 2004) or the logistic equation (Parvinen and Egas 2004; de Mazancourt and Dieckmann 2004) to describe habitat-specific population growth. In these models habitat specialization is subject to a tradeoff, such that a high value in the carrying capacity or the intrinsic growth rate in one habitat is bought at the expense of a low carrying capacity or growth rate in the other habitat. Evolution and coexistence are then studied by assuming variation in the degree of habitat-specialization in terms of either $r$ or $K$. These parameters have an interpretation only at the level of populations while mutations causing evolutionary change occur at the level of the individual. Therefore the models mentioned above employ a shortcut because a description of how variation at the level of the individual is linked to variation in population-level parameters is skipped. Such a shortcut is only permissible when the assumed patterns of variation at the level of the demographic parameters can be derived from variation in underlying individual-level traits, an issue that can only be evaluated with models that are based on an explicit mapping from processes at the level of individuals to population-level parameters.

Although our study is motivated by the specific problem introduced above, the issue at hand is of a much more general nature. Genetic variation occurs at the level of DNA sequences and ultimately affects the make-up of a population. The step from individual sequences to population-level characteristics can be described by a cascade of mappings. Genotypes are mapped to enzymes and their regulation. During development these characteristics might be mapped to, e.g., morphometrical properties of the beak of a bird, which determine its handling times for different seeds, which determines energy uptake, which determines offspring production, which determines population growth rate. The general question then becomes how patterns of variation and covariation at one level are mapped to the next level.

The aim of this paper is to show how an explicit mapping from individual-level traits to population-level parameters can help us to understand how evolution is likely to work by informing us about how patterns of variation and covariation at lower levels can constrain resulting patterns of variation at higher levels. In the following section we discuss two pitfalls that con occur when an explicit description of the mapping from individual-level traits to demographic parameters is skipped. We then continue by illustrating these issues by means of two examples and conclude by sketching how our viewpoint affects the results of the above mentioned studies. 


\section{Population-Level Parameters Derive From Underlying Traits at the Level of the Individual}

In this section we use the logistic equation to illustrate two separate issues that emerge when patterns of variation and covariation at the level of population-level demographic parameters are derived from variation in underlying individual-level traits.

The logistic equation is given by

$$
\frac{1}{N} \frac{\mathrm{d} N}{\mathrm{~d} t}=r\left(1-\frac{N}{K}\right)
$$

where $N$ denotes population density, $r$ the intrinsic rate of increase and $K$ the carrying capacity. The logistic equation is widely used to describe density-dependent population growth of a single species with only two parameters. The fact that $r$ and $K$ do not have an interpretation at the level of the individual has triggered a series of derivations from first principles at the level of the individual. Such derivations either assume density dependence in the birth rate $b$ (MacArthur (1972, p. 56), Schoener (1973), Edelstein-Keshet (1988, p. 119), see also Kooi et al. (1998)) or in the death rate $d$ (Gyllenberg 2005). For derivations that do not describe the dynamics of individuals but the fraction of either inhabited patches in a meta-population or of infected individuals in a population of susceptible and infected individuals see Hanski (1999, p. 56) and Diekmann and Heesterbeek (2000, p. 212), respectively. The derivations that assume a density-dependent birth rate have a limited scope because they predict a negative birth rate when the population size passes some threshold and we do not consider these derivations any further. All derivations that assume a density-dependent death rate implement the idea that the death rate is linearly increasing with population density:

$$
\frac{1}{N} \frac{\mathrm{d} N}{\mathrm{~d} t}=b-(d+\alpha N)=(b-d)\left(1-\frac{\alpha}{b-d} N\right),
$$

where the biological interpretation of the positive proportionality constant $\alpha$ depends on the specific derivation. From comparing equation (2) with equation (1) we see that $r=b-d$ and $K=(b-d) / \alpha$.

Based on these derivations two observations can be made. Firstly, $K$ depends linearly on those traits that underly $r$ and any variation that affects $r$ causes $K$ to vary proportionally. Thus, seemingly independent parameters at one level are not necessarily independent when they are derived from an underlying level. A conclusion from the above derivation is that models that use the logistic equation as a building block and that assume variation in $r$ while $K$ is kept constant (e.g. Parvinen and Egas 2004) do not have an interpretation at the level of the individual. We do not claim that it is impossible to construct a derivation of the logistic equation from first principles such that a individual-level trait exists that contributes to $r$ but not to $K$. But even in this case independent variation of $r$ can only be motivated if genetic variation is likely to contribute only to those traits that do not affect $K$.

Secondly, $K$ can change independently of $r$ only through a change in $\alpha$. This parameter is

inversely related to $K$, hence, any change in $\alpha$ affects $K$ in a non-linear way. This observation 
becomes important when the evolutionary dynamics of correlated traits is studied. Assume a situation where an organism occupies two different habitats that require different adaptations and that population growth in each habitat can be described by the logistic equation (eq. 2). In this situation it is likely that a constraint exists that prevents a genotype from being optimally adapted to both habitats simultaneously. Here we adopt the viewpoint that such a constraint can be visualized as the outer boundary of the set of possible phenotypes to which we will refer as trade-off curve (fig. 1). Assume further that adaptations to the habitats occur in the traits $\alpha_{1}$ and $\alpha_{2}$. Since population growth is increasing with decreasing values of $\alpha_{i}$ (eq. 2), selection will push a population's trait distribution from high values of $\alpha_{i}$ towards lower values until the trade-off is hit. From then onward selection will keep the phenotype distribution close to the trade-off relative to the mutational step size. Patterns of covariation within a population will then depend on the shape of the outer boundary of the set of possible phenotypes and different trade-off curves correspond to different such boundaries. Arnold (1992) refers to this scenario as the Charnov-Charlesworth model for equilibrium genetic covariance for a single pair of traits (cf. Charnov 1989; Charlesworth 1990). The pattern of covariation in individual-level traits causes specific patterns of covariation at the level of demographic parameters (fig. 1). In our example phenotypes are mapped from the $\left(\alpha_{1}, \alpha_{2}\right)$-space to the $\left(K_{1}, K_{2}\right)$-space by $\left(K_{1}, K_{2}\right)=\left(\left(b_{1}-d_{1}\right) / \alpha_{1},\left(b_{2}-d_{2}\right) / \alpha_{2}\right)$. In the same way we can map the trade-off curve from one level to the next. The result of this mapping is shown in figure 1. Note that the phenotype distribution in the $\left(K_{1}, K_{2}\right)$-space lies below and to the left of the trade-off because population growth is increasing with increasing values of $K_{i}$.

The important message from this example is that properties of the phenotype distribution and the trade-off are not necessarily conserved when mapped from one level to the other. The phenotypes indicated by the dots in the $\left(\alpha_{1}, \alpha_{2}\right)$-space in figure 1 show relative low values of $\alpha_{i}$ for both habitats. The corresponding trade-off is called "weak" because the habitat generalist is only slightly inferior in each habitat when compared to the habitat specialists. On the other hand, phenotypes in the $\left(K_{1}, K_{2}\right)$-space show relative low carrying capacities in both habitats. In this case the the trade-off is called "strong" because habitat generalists are strongly inferior in each habitat in terms of their carrying capacity when compared to the corresponding specialist. From this observation two questions emerge. Firstly, can an a priori chosen curve for the trade-off between $K_{1}$ and $K_{2}$ be derived from a curve in the underlying constraint that connects $\alpha_{1}$ and $\alpha_{2}$ ? Secondly, assume that the answer to the first question is 'yes', how biologically plausible is it to assume the type of curve for the trade-off between $\alpha_{1}$ and $\alpha_{2}$ that maps to the a priori chosen curve for the trade-off between $K_{1}$ and $K_{2}$ ? In the following examples we will show that in some cases a weak trade-off between habitat-specific carrying capacities cannot be derived from any underlying trade-off and that in other cases specific curves for the trade-off at the higher level can only be realized by assuming rather extreme curvatures for the trade-off at the underlying level.

Before we continue to illustrate our point we want to point out that the very same two observations can be made for the Ricker equation $N_{t+1} / N_{t}=\exp \left[r\left(1-N_{t} / K\right)\right]$. For derivations of the Ricker equation from first principles see Royama (1992), Gatto (1993), Gurney and Nisbet (1998), 
Sumpter and Broomhead (2001), Thieme (2003), Geritz and Kisdi (2004), and Brännström and Sumpter (2005).

\section{Two Examples}

We want to illustrate by means of two examples how patterns of covariation between demographic parameters can be derived from an explicit model of individual-level processes that contribute to the demographic parameters. Our examples are strongly inspired by the models of Wilson and Yoshimura (1994), Egas et al. (2004), Parvinen and Egas (2004) and de Mazancourt and Dieckmann (2004). In this section we develop a two-habitat version of the logistic and the Ricker equation from first principles. This provides us with explicit expressions for the carrying capacity in terms of individual-level traits. We then investigate how specific trade-off curvatures underlying traits that contribute to carrying capacity affect the curvature of the resulting trade-off between habitat specific carrying capacities. These results are shown in the following section. In the final section before the discussion we show how evolutionary predictions can differ when evolutionary change is directly assumed in the carrying capacities when compared to trade-offs in underlying individual-level traits.

\section{Continuous Time: Fast Migration Between Habitats}

Assume that individuals migrate at a high rate between habitats and that travel time is negligible. This means that the environment is fine-grained. Under the assumption that birth and death rates are small compared to the migration rates, we calculate the probability for an individual to be in habitat one, $p_{1}$, or to be in habitat two, $p_{2}=1-p_{1}$ in appendix A. Furthermore, we assume that birth and death processes are determined instantaneously, that is, whether an individual dies or gives birth at a certain moment in time, purely depends on its current habitat. If population growth within each habitat can be described by the logistic equation (eq. 1), then the population dynamics is given by

$$
\frac{\mathrm{d} N}{\mathrm{~d} t}=N\left[p_{1} r_{1}\left(1-\frac{p_{1} N}{K_{1}}\right)+p_{2} r_{2}\left(1-\frac{p_{2} N}{K_{2}}\right)\right]
$$

with $r_{i}$ and $K_{i}$ the habitat-specific intrinsic growth rates and carrying capacities, respectively. The dynamics has a single, nontrivial, stable equilibrium at

$$
\hat{N}=\frac{\left(p_{1} r_{1}+p_{2} r_{2}\right) K_{1} K_{2}}{p_{1}^{2} r_{1} K_{2}+p_{2}^{2} r_{2} K_{1}}
$$

In order to give the parameters $r_{i}$ and $K_{i}$ an interpretation at the level of the individual, we present in appendix A one possible derivation of equation (3) based on individual-level processes. This derivation is inspired by Royama's derivation of the Ricker equation (1992, pp. 144, see

following section). We assume that individuals die at some minimum rate when they can exploit a 
competition neighborhood of size $a$ on their own. The size of this neighborhood is determined by a variety of biological properties like the conversion efficiency of food into energy or the efficiency with which resources are gathered. The minimum death rate increases incrementally by $l$, a positive constant, with each additional conspecific that enters the competition neighborhood of the focal individual. For a single habitat this mechanism yields

$$
\frac{\mathrm{d} N}{\mathrm{~d} t}=N(b-d)\left(1-\frac{l a}{b-d} N\right)
$$

where $b$ and $d$ are the density independent birth and death rate, respectively. Equation (5) corresponds to equation (2), but now the demographic parameters $r$ and $K$ have an interpretation in terms of individual-level traits: $r=b-d$ and $K=(b-d) / l a$. This description holds for each habitat so that $r_{i}=b_{i}-d_{i}$ and $K_{i}=\left(b_{i}-d_{i}\right) / l_{i} a_{i}$. If we insert these descriptions into equation (3), we get the following two-habitat version:

$$
\frac{\mathrm{d} N}{\mathrm{~d} t}=N\left[p_{1}\left(b_{1}-d_{1}\right)\left(1-\frac{l_{1} a_{1}}{b_{1}-d_{1}} p_{1} N\right)+p_{2}\left(b_{2}-d_{2}\right)\left(1-\frac{l_{2} a_{2}}{b_{2}-d_{2}} p_{2} N\right)\right] .
$$

\section{Discrete Time: Juvenile Dispersal}

Here we assume that generations are discrete and non-overlapping. At birth individuals are randomly distributed over two habitats with probabilities $p_{1}$ and $p_{2}=1-p_{1}$. These probabilities

are proportional to the relative size of the two habitats and independent of the habitat of birth. The latter is the case either because of very effective dispersal or due to a fine-grained environment. Once settled individuals stay within their habitat until death. In case of a fine-grained environment this implies that organisms are sessile. If we assume that the population dynamics within each habitat can be described by the Ricker equation (Ricker 1952), then population growth is given by:

$$
N_{t+1}=N_{t}\left[p_{1} \exp \left(r_{1}\left(1-\frac{p_{1} N_{t}}{K_{1}}\right)\right)+p_{2} \exp \left(r_{2}\left(1-\frac{p_{2} N_{t}}{K_{2}}\right)\right)\right] .
$$

Only in case of symmetric parameter values $r_{1}=r=r_{2}, K_{1}=K=K_{2}$ and $p_{1}=0.5=p_{2}$ can we calculate the equilibrium population size analytically as $\hat{N}=2 K$. The population dynamics of this case are well understood. The equilibrium $\hat{N}=2 K$ is stable for $r<2$, larger value of $r$ lead to cycles and eventually chaotic dynamics (May and Oster 1976). Here we restrict ourselves to parameter values that produce stable equilibria.

In order to get an individual-level interpretation of equation (7) we again follow Royama (1992, pp. 144). Royama assumes that the offspring number an individual produces, $R_{n}$, decreases exponentially with the number of conspecifics $n$ within a competition neighborhood of size $a$ : $R_{n}=R_{0} k^{n}$. The sensitivity parameter $k$ is a positive constant smaller than one. For the case that individuals are Poisson distributed over the habitat, Royama (1992) shows that

$$
N_{t+1}=N_{t} \exp \left(\log R_{0}\left(1-\frac{a(1-k)}{\log R_{0}} N_{t}\right)\right) \text {. }
$$


This is the Ricker equation where $r=\log R_{0}$ and $K=\log R_{0} /(a(1-k))$. By combining equation (8) with equation (7), we get a two-habitat version of the Ricker equation:

$$
\begin{aligned}
N_{t+1}=N_{t} & {\left[p_{1} \exp \left(\log R_{01}\left(1-\frac{a_{1}\left(1-k_{1}\right)}{\log R_{01}} p_{1} N_{t}\right)\right)\right.} \\
& \left.+p_{2} \exp \left(\log R_{02}\left(1-\frac{a_{2}\left(1-k_{2}\right)}{\log R_{02}} p_{2} N_{t}\right)\right)\right]
\end{aligned}
$$

\section{The Mapping of Trade-Offs}

The preceding section provides us with an individual-based interpretation the population-level parameters $K_{1}$ and $K_{2}$ :

$$
\begin{aligned}
K_{i}=\frac{b_{i}-d_{i}}{a_{i} l_{i}} & r_{i}=b_{i}-d_{i} & \text { logistic equation } \\
K_{i}=\frac{\log R_{0 i}}{a_{i}\left(1-k_{i}\right)} & r_{i}=\log R_{0 i} & \text { Ricker equation. }
\end{aligned}
$$

We now investigate how a trade-off between the habitat specific carrying capacities can be derived from a trade-off in traits at the level of the individual. Here we assume that mutational change occurs for the size of the competition neighborhoods $a_{1}$ and $a_{2}$ and that these traits are coupled by a trade-off, which can be written as a function $a_{2}\left(a_{1}\right)$ with $\mathrm{d} a_{2} / \mathrm{d} a_{1}<0$. All other traits are assumed to be fixed parameters. We choose $a_{1}$ and $a_{2}$ because they can, at least in principle, be measured at the level of the individual and because they influence the carrying capacities in the logistic and the Ricker equation in the same way, which facilitates a comparison between these models. From now on we refer to a trade-off between $a_{1}$ and $a_{2}$ as a trade-off in $\boldsymbol{a}$, and similarly, to a trade-off between $K_{1}$ and $K_{2}$ as a trade-off in $\boldsymbol{K}$.

Next we introduce specific trade-off parameterizations for $a_{2}\left(a_{1}\right)$ and $K_{2}\left(K_{1}\right)$. This allows us to visualize how a trade-off in $\boldsymbol{a}$ maps onto a trade-off in $\boldsymbol{K}$, or, vice versa, what trade-off in $\boldsymbol{a}$ is implicitly assumed when a specific trade-off in $\boldsymbol{K}$ is chosen. Our parameterizations generalize the trade-off function used by Egas et al. (2004) and Parvinen and Egas (2004).

$$
\begin{gathered}
\boldsymbol{a}=\left(a_{1}, a_{2}\right)=\left(a_{1 \max }-a_{1 \operatorname{var}}(1-\theta)^{\frac{1}{z}}, a_{2 \max }-a_{2 \operatorname{var}} \theta^{\frac{1}{z}}\right) \\
\boldsymbol{K}=\left(K_{1}, K_{2}\right)=\left(K_{1 \min }+K_{1 \operatorname{var}}(1-\theta)^{\frac{1}{z}}, K_{2 \min }+K_{2 \operatorname{var}} \theta^{\frac{1}{z}}\right)
\end{gathered}
$$

Here $a_{1 \mathrm{max}}, a_{1 \mathrm{var}}, a_{2 \mathrm{max}}, a_{2 \mathrm{var}}, K_{1 \mathrm{~min}}, K_{1 \mathrm{var}}, K_{2 \mathrm{~min}}, K_{2 \mathrm{var}}$ are positive constants determining the range of possible parameter values while the positive parameter $z$ determines the curvature of the trade-off curve.

Note the following difference between the two trade-off parameterizations. In equation (12) 
$\theta=0$ corresponds to a low value of $a_{1}$ and to a high value of $a_{2}$. In equation (13) the opposite holds true, $\theta=0$ corresponds to a high value of $K_{1}$ and to a low value of $K_{2}$. Since population growth is decreasing in $a_{i}$ (eq. 6 and eq. 9 ) but increasing in $K_{i}$ (eq. 3 and eq. 7 ) this means that in both cases $\theta=0$ corresponds to a specialist for habitat one while $\theta=1$ corresponds to a specialist for habitat two. A similar pattern applies to the curvature of the trade-off and the corresponding curvature parameter $z$ (fig. 2). Values of $z<1$ correspond to a concave trade-off in $\boldsymbol{a}\left(\mathrm{d}^{2} a_{2} / \mathrm{d} a_{1}^{2}<0\right)$ and to a convex trade-off in $\boldsymbol{K}\left(\mathrm{d}^{2} K_{2} / \mathrm{d} K_{1}^{2}>0\right)$. The opposite pattern holds for $z>1$. Hence, these parameterizations are such that $z<1$ corresponds to a strong trade-off and $z>1$ corresponds to a weak trade-off in both $\boldsymbol{a}$ and $\boldsymbol{K}$. This terminology is motivated by the following observation. In case of symmetric values for $a_{i \max }, a_{i \mathrm{var}}, K_{i \mathrm{~min}}$, and $K_{i \mathrm{var}}$ all phenotypes that lie on a linear trade-off $(z=1)$ have exactly the same value for $a$ and $K$, respectively, when averaged over both habitats. On a convex trade-off a generalist has a lower competition neighborhood size (eq. 12) and a lower carrying capacity (eq. 13) than a specialist when averaged over both habitats. Such a generalist is superior over the specialists in terms of its average competition neighborhood size but inferior in terms of its average carrying capacity. The opposite pattern holds for concave trade-offs.

How does the function $a_{2}\left(a_{1}\right)$ mediates the trade-off in $\boldsymbol{K}$ ? To see this we have to combine equation (10) and (12). The result is shown in figure 2a\&b. Note that the curvature of the derived trade-off in $\boldsymbol{K}$ can change from convex to concave along a single trade-off curve (see fig. $2 \mathrm{~b}$, right panel). In the following we refer to the curvature around the generalist's trait with $\theta=0.5$. In figure $2 \mathrm{a}$ the trait space encompasses values of $a_{i}$ between 0.1 and $0.2\left(a_{i \max }=0.2, a_{i \mathrm{var}}=0.1\right)$ while in figure $2 \mathrm{~b}$ the trait space encompasses values between 0.1 and $1\left(a_{i \max }=1, a_{i \mathrm{var}}=0.9\right)$. In the first case all trade-offs in $\boldsymbol{a}$ corresponding to $z<2$ are mapped into a strong trade-off in $\boldsymbol{K}$. In other words, for trade-offs in $\boldsymbol{a}$ with $1<z<2$, that is, for moderately weak trade-offs, their weakness is not inherited. In the second case, only extremely weak trade-offs in $\boldsymbol{a}$ corresponding to $z>\approx 12$ are mapped onto weak trade-offs in $\boldsymbol{K}$. For trade-offs with $1<z<12$, the weakness is not inherited. Hence, weak trade-offs in $\boldsymbol{a}$ are mapped to strong trade-offs in $\boldsymbol{K}$ for $z$-values below some threshold. In appendix B we show that this threshold increases with increasing values of $a_{i \mathrm{var}}$. In the limit of $a_{i \mathrm{var}}=0$ the threshold becomes one and the curvature property is always

inherited. In the limit of $a_{i \text { var }}=\infty$ the threshold becomes infinity, hence, any trade-off in $\boldsymbol{a}$, weak or strong, is mapped to a strong trade-off in $\boldsymbol{K}$.

Models assuming a trade-off directly at the level of the carrying capacities make an implicit assumption about the shape of the trade-off in an individual-level trait. This implicit assumption can be laid bare by applying the inverse mapping, from the trade-off in the carrying capacities $\boldsymbol{K}$ to the trade-off in $\boldsymbol{a}$. The result of this exercise is shown in figure $2 c \& d$.

\section{Evolutionary Dynamics}

Analyses of long term evolution should be based on an invasion argument: Can a rare mutant type increase in frequency or is it doomed to extinction? This is determined by its invasion fitness 
$s$, its long term average growth rate when rare in an environment determined by a given resident type (e.g., Metz et al. 1992). If $s>0$, then such a mutant has a positive probability to invade the resident population and if $s<0$ such a mutant will disappear. Under certain conditions an invasion analysis is equivalent to solving an optimization criterion (Metz et al. 1996). The quantity that is maximized by evolution cannot be chosen a priori but has to be derived from an invasion argument (Mylius and Diekmann 1995). For our examples we are able to show that equilibrium population size $\hat{N}$ serves as an optimization criterion. Populations with trait values that correspond to a higher equilibrium population size $\hat{N}$ replace populations with lower equilibrium population size and and trait values that maximize population size are potential endpoints of evolution. These trait values are both attractors of the evolutionary dynamics and are uninvadable by individuals with other trait values. Eshel (1983) coined the term continuously stable strategies (CSSs) for such trait values. Trait values that correspond to minima of $\hat{N}$ are evolutionary repellors from where the evolutionary dynamics moves away. The existence of an optimization criterion allows us to visualize the evolutionary process using Levins' fitness set approach (Levins 1962; Rueffler et al. 2004). In this graphical method the trade-off curve is plotted on top of the contour lines of the fitness landscape given by $\hat{N}$ (fig. 4). Evolutionary endpoints are those phenotypes on the trade-off curve that lie on the highest fitness contour line.

\section{Fast Migration Between Habitats}

First we consider the case where evolutionary change is assumed to directly affect $K_{1}$ and $K_{2}$. Invasion fitness of a mutant type $\boldsymbol{K}^{\prime}=\left(K_{1}^{\prime}, K_{2}^{\prime}\right)$ in a resident population with carrying capacities $\boldsymbol{K}=\left(K_{1}, K_{2}\right)$ can be derived from equation (3) as

$$
s\left(\boldsymbol{K}^{\prime}, \boldsymbol{K}\right)=p_{1} r_{1}+p_{2} r_{2}-\hat{N}(\boldsymbol{K})\left(\frac{p_{1}^{2} r_{1}}{K_{1}^{\prime}}+\frac{p_{2}^{2} r_{2}}{K_{2}^{\prime}}\right) .
$$

Equation (14) is obviously monotonically decreasing in $\hat{N}$ (eq. 4). As mentioned above, this is a sufficient condition for the equilibrium population size to be an optimization criterion (Mylius and Diekmann 1995). Figure 4a shows the numerically calculated location of the minima and maxima of the optimization criterion as a function of the curvature parameter $z$ for one specific set of symmetric parameter values. For weak trade-offs the habitat generalist with $K_{1}=K_{2}$ maximizes equilibrium population size $\hat{N}$ and is therefore a CSS. The habitat generalist remains to be a CSS for moderately strong trade-offs and it is only for very strong trade-offs with $z<0.35$ that the generalist turns into an evolutionary repellor (fig. 4).

In appendix $\mathrm{C}$ we show that whether the habitat generalist with $K_{1}=K_{2}$ constitutes a

minimum or a maximum of $\hat{N}$ also depends on the parameters $K_{i \min }$ and $K_{i \text { var }}$, i.e., on the range of possible parameter values for $K_{i}$. Small values of $K_{\text {imin }}$ favor the generalist even for $z<1$ (strong trade-offs) while with small values of $K_{i \text { var }}$ a situation is approached where weak trade-offs select for a generalist while strong trade-offs select for specialists.

Does the evolutionary dynamics change when we implement evolutionary change in individual- 
level traits? Invasion fitness of a rare mutant type with competition neighborhood size $\boldsymbol{a}^{\prime}=\left(a_{1}^{\prime}, a_{2}^{\prime}\right)$ in a population with $\boldsymbol{a}=\left(a_{1}, a_{2}\right)$ can be derived from equation (6) as

$$
s\left(\boldsymbol{a}^{\prime}, \boldsymbol{a}\right)=p_{1} r_{1}+p_{2} r_{2}-\hat{N}(\boldsymbol{a})\left(p_{1}^{2} a_{1}^{\prime} l_{1}+p_{2}^{2} a_{2}^{\prime} l_{2}\right),
$$

where $r_{i}=b_{i}-d_{i}$. As in the previous version, fitness is a monotonically decreasing function of $\hat{N}$, which therefore qualifies as a optimization criterion. From equation (4) we can derive that $\hat{N}=\left(p_{1} r_{1}+p_{2} r_{2}\right) /\left(p_{1}^{2} a_{1} l_{1}+p_{2}^{2} a_{2} l_{2}\right)$. Differentiation of $\hat{N}$ reveals that critical points are given by $\mathrm{d} a_{2} / \mathrm{d} a_{1}=-p_{1}^{2} l_{1} /\left(p_{2}^{2} l_{2}\right)$ and that the sign of the second derivative equals the sign of $-\mathrm{d}^{2} a_{2} / \mathrm{d} a_{1}^{2}$. Hence, critical points of $\hat{N}$ are maxima for $z>1$ (weak trade-offs) and minima for $z<1$ (strong trade-offs). For $z=1$ all trait combinations are selectively neutral. Figure $4 \mathrm{~b}$ depicts this results for the case of symmetric parameter values. A comparison of figure $3 \mathrm{a}$ and $3 \mathrm{~b}$ shows that the range of $z$-values that favor habitat generalists over specialists is considerably smaller when the trade-off is implemented between $a_{1}$ and $a_{2}$. This indicates that in this case the shortcut of directly implementing a trade-off in demographic parameters overestimates the likelihood of finding generalists.

It is illuminating to plot these results in terms of fitness sets (Levins 1962). The fitness landscape, that is, the optimization criterion, can be plotted either as a function of the demographic parameters $K_{1}$ and $K_{2}$ (fig. $4 \mathrm{a} \& \mathrm{c}$ ) or as a function of the underlying individual-level traits $a_{1}$ and $a_{2}$ (fig. 4b). In the first case the contours of the fitness landscape are given by those values of $K_{1}$ and $K_{2}$ that result in equal values $c$ of the optimization criterion $\hat{N}$. These $K_{i}$-values lie on convex hyperbolas given by $K_{2}=c p_{2}^{2} r_{2} K_{1} /\left(K_{1}-c p_{1}^{2} r_{1}\right)$. In the second case the contours of the fitness landscape are straight lines with a negative slope given by $a_{2}=\left(1-c p_{1}^{2} a_{1} l_{1}\right) /\left(c p_{2}^{2} l_{2}\right)$. In a next step we plot different trade-off curves on top of the contour plot of the fitness landscape. In figure 4a this is done for trade-offs in $\boldsymbol{K}$ and in $4 \mathrm{~b}$ for trade-offs in $\boldsymbol{a}$. In figure 4c this is done again for a trade-off in $\boldsymbol{K}$, but now the trade-off is derived from the underlying trade-off in $\boldsymbol{a}$. From figure $4 \mathrm{a}$ we see that fitness landscapes with convex fitness contours favor generalists and it is only for very strong trade-offs (e.g., $z=1 / 4$ ) that specialists do better (cf. fig. 3a). In the presence of a fitness landscape with linear contours (fig. 4b) weak trade-offs favor generalists and strong trade-offs favor specialists (cf. fig. 3b). Figure 4c shows that optimization in terms of $K$ and $a$ are equivalent approaches as long as the trade-off in $\boldsymbol{K}$ is derived from an underlying trade-off in $\boldsymbol{a}$. In both case the trade-off curve given by $z=1$ exactly follows a contour line of the fitness landscape which accounts for the evolutionary neutrality of all traits.

\section{Juvenile Dispersal}

Fitness in discrete time models can be expressed more easily as $w=\exp (s)$. If $w>1$, a mutant is able to invade while a mutant with $w<1$ cannot invade. As in the previous section we first perform an evolutionary analysis under the assumption that $K_{1}$ and $K_{2}$ are traded-off directly followed by an analysis where evolutionary change is implemented in the size of the two competition neighborhoods $a_{1}$ and $a_{2}$. In both cases the fitness function is monotonically decreasing in $\hat{N}$, which therefore again qualifies as an optimization criterion (Mylius and Diekmann 1995). 
Unfortunately, we can only calculate $\hat{N}$ analytically for the case of symmetric parameter values and a consumer that is equally specialized for both habitats. Hence, the maximization of $\hat{N}$ has to be done numerically.

For the first case where the trade-off is directly assumed in $\boldsymbol{K}$ we find the following fitness function:

$$
w\left(\boldsymbol{K}^{\prime}, \boldsymbol{K}\right)=p_{1} \exp \left(r_{1}\left(1-\frac{p_{1} \hat{N}(\boldsymbol{K})}{K_{1}^{\prime}}\right)\right)+p_{2} \exp \left(r_{2}\left(1-\frac{p_{2} \hat{N}(\boldsymbol{K})}{K_{2}^{\prime}}\right)\right) .
$$

Numerical analysis of the optimization criterion $\hat{N}$ reveals a pitchfork bifurcation of $\theta$-values that correspond to extrema in $\hat{N}$ (fig. 3c). For small values of $z$ (strong trade-offs) the generalist is an evolutionary repellor. The generalist turns into a CSS when some threshold value of $z$ is passed. For $r<2$, i.e., for stable population dynamical equilibria, we prove in appendix $\mathrm{C}$ that this threshold always has a value smaller than one.

In the second case where the trade-off is assumed between the underlying traits $a_{1}$ and $a_{2}$ invasion fitness is given by

$$
w\left(\boldsymbol{a}^{\prime}, \boldsymbol{a}\right)=p_{1} \exp \left[r_{1}\left(1-\frac{p_{1} \hat{N}(\boldsymbol{a}) a_{1}^{\prime}\left(1-k_{1}\right)}{r_{1}}\right)\right]+p_{2} \exp \left[r_{2}\left(1-\frac{p_{2} \hat{N}(\boldsymbol{a}) a_{2}^{\prime}\left(1-k_{2}\right)}{r_{2}}\right)\right] .
$$

By numerical calculations we find again a pitchfork bifurcation (fig. 3d). For this case we can prove (app. C) that the change from a repelling generalist strategy to a generalist which is a CSS takes place for some $z$-value larger than one (for a weak trade-off). In appendix $\mathrm{C}$ we also show that the bifurcation point moves towards higher $z$-values with increasing population growth rate $r$. This means that fast growth favors habitat specialization. Figure $4 \mathrm{~d}-\mathrm{f}$ illustrate these results with the use of fitness sets. A comparison of figure $3 \mathrm{c}$ and $3 \mathrm{~d}$ shows that our approach of implementing the trade-off at the individual level results in a even smaller range of $z$-values that favor habitat generalists than in the previous example.

\section{Discussion}

Genetic variation is the fuel for evolutionary change. This variation occurs at the level of DNA sequences. The direction of evolutionary change depends on the available genetic variation and on the per capita growth rate of the different genotypes in the environment where selection takes place. A complete understanding of the evolutionary process would require knowledge of how variation at the level of the DNA sequence is mapped through the process of development to variation in demographic parameters. In most cases we are far away from such a detailed knowledge and in order to deal with this difficulty theoretical biologists have followed either of two different roads. Theoretical population geneticists study variation in allele space and assume an extremely simplified genotype-phenotype map by assigning fixed fitness values to alleles. The other approach neglects the genotype-phenotype map altogether and studies the effect of variation directly at the level of phenotypes (optimization models, quantitative genetics, game theory, and 
adaptive dynamics). Either approach is a shortcut and it is important to develop an understanding of the possible consequences of such shortcuts. In this paper we show how patterns of variation and covariation at one level are mapped to a higher level and pinpoint two pitfalls that can occur when variation at a higher level is not derived from variation in underlying traits.

Our paper is motivated by a recent series of models where habitat specific population growth is described by either the logistic equation (Parvinen and Egas 2004; de Mazancourt and Dieckmann 2004) or the Ricker equation (Wilson and Yoshimura 1994; Egas et al. 2004). In these models variation is implemented in habitat specific growth rates or carrying capacities. Assuming evolutionary change in these population-level parameters might not be admissible for at least two reasons. Firstly, population-level parameters that appear as independent variables in a population dynamical equation might not be independent when derived from processes at the individual level. Such is the case with $r$ and $K$ in both the logistic and the Ricker equation. In these growth models $K$ appears to be linearly dependent on $r$. Consequently, variation in $r$ alone cannot be derived from variation in an underlying trait. Independence of $r$ and $K$ should only be assumed based on a derivation showing how different independent traits at the individual level can affect the different demographic parameters separately. Kuno (1991), Olson (1992), and Berryman (1992) all discuss why the logistic equation is likely to be a bad candidate to find such a derivation. Secondly, tradeoffs between population-level parameters originate from correlations in underlying individual-level traits. If the mapping from these traits to higher level parameters is nonlinear, then specific tradeoff curvatures are not inherited from one level to the next. By implementing a certain trade-off curvature between population-level parameters the modeler makes an implicit assumption about the nature of the trade-off at the individual-level. Because of the involved nonlinearity this assumption might often be rather unrealistic and in some cases, as we have shown, a certain curvature cannot be derived from an underlying trade-off at all.

The observation that, based on all derivations of the logistic equation and the Ricker equation known to us, the carrying capacity increases linearly with intrinsic growth rate, has important consequences for the theory of $r$ - and $K$-selection. In the classical sense, as popularized by MacArthur and Wilson (1967), Roughgarden (1971) and Charlesworth (1971), this theory states that variable environments where population densities are regularly set back to low values, select genotypes with high intrinsic growth rates while more stable environments select genotypes corresponding to high equilibrium population densities. The influential paper by Roughgarden (1971) uses the logistic equation to derive these predictions formally. In this model a trade-off is assumed: genotypes with a high intrinsic growth rate correspond to a low carrying capacity and vice versa. This assumption is clearly at odds with the viewpoint championed in this paper suggesting that $r$ and $K$ are positively rather than negatively correlated. Thus, in models that are based on the logistic equation but where the demographic parameters are derived from processes at the level of the individual, selection for high growth rates result in a concomitant increase of the carrying capacity. This point has also been made by Kuno (1991). That the theory of $r$ - and $K$-selection in its narrow sense is flawed for other reasons is known at least since the important paper by Matessi and Gatto (1984). They have 
shown that stable environments need not select the genotype corresponding to the highest carrying capacity but rather select the genotype that can live on the fewest resources. These authors suggest to use the terms $r$ - and $K$-selection to refer to the conditions of selection (density independent vs density dependent) rather than to the outcome of selection ( $r$-maximization vs $K$-maximization).

\section{Application to the Recent Literature}

The ideas presented in this paper affect the results of a recent series of papers that study the evolution of habitat specialization. In these models mutational change is assumed for habitatspecific carrying capacities or intrinsic growth rates.

Wilson and Yoshimura (1994) have explored the scope for coexistence of two habitat specialists and a habitat generalist as determined by their habitat specific carrying capacities in a model where habitat specific growth is described by the Ricker equation. In their basic model version the carrying capacity for a specialist is ten times higher in the habitat it is adapted to when compared to the habitat it is not adapted to. This situation corresponds to our figure $2 \mathrm{~b} \& \mathrm{~d}$, where the two specialists are characterized by $\boldsymbol{K}=(1,0.1)$ and $\boldsymbol{K}=(0.1,1)$. The shape of the trade-off is determined by the carrying capacities of the generalist. To cover trade-off relations from strong to weak, Wilson and Yoshimura varied the carrying capacity of the generalist between 0.3 and 0.99 . Wilson and Yoshimura (1994) find that coexistence is possible for generalists ranging from $\boldsymbol{K}=(0.99,0.99)$ to $\boldsymbol{K}=(0.4,0.4)$. According to our parameterization (see eq. [13]), these generalists correspond to trade-offs in $\boldsymbol{a}$ that are parameterized by values between $z=617$ (for $\boldsymbol{K}=(0.99,0.99)$ ) and $z=3.8$ (for $\boldsymbol{K}=(0.4,0.4)$ ). Hence, all these trade-offs correspond to very weak or extremely weak trade-offs in $\boldsymbol{a}$ (cf. fig. 2d). Moderately weak, linear and strong trade-offs in $\boldsymbol{a}$ all correspond to strong trade-offs in $\boldsymbol{K}$ (cf. fig. $2 \mathrm{~b}$ ) and do not allow for the coexistence of two specialists and a generalist. From this viewpoint the scope for coexistence seems to be far more restricted than suggested by Wilson and Yoshimura.

Egas et al. (2004) present a re-analysis of the model of Wilson and Yoshimura. In one version of their model they assume that the habitat-specific carrying capacities can vary continuously between zero and 100. A carrying capacity of zero corresponds to an infinitely large competition neighborhood size with $a_{i \max }=\infty=a_{i v a r}$. In appendix B we prove that in this case any trade-off in $\boldsymbol{a}$ is mapped into a strong trade-off in $\boldsymbol{K}$. However, from the analysis in Egas et al. (2004) it becomes clear that coexistence of two specialists and a generalists requires a weak trade-off in $\boldsymbol{K}$ in all cases where environmental variability is not extremely high. This perspective therefore suggests again that coexistence is far more restricted than it appears from the analysis of the authors.

Parvinen and Egas (2004) studied the evolution of habitat specialization in a metapopulation model with two types of habitat and logistic growth within patches. Evolutionary change is assumed in either habitat-specific intrinsic growth rates or carrying capacities. Currently no derivation of the logistic equation known to us provides a mechanism that would allow to vary the intrinsic growth rate while leaving the carrying capacities constant: in all published mechanisms known to 
us the latter is linearly dependent on the former. Hence, we lack an individual-based interpretation of habitat specialization in terms of intrinsic growth rates.

The main purpose of a paper by de Mazancourt and Dieckmann (2004) is to extend Levins' (1962) graphical fitness set approach to accommodate frequency-dependent selection. In order to illustrate their methodology they analyze a model of one consumer feeding in two habitats. The consumer grows logistically in each habitat and evolves in a trait that determines habitat-specific carrying capacities. Evolution in the carrying capacities is assumed to be constrained by a trade-off and in the specific case analyzed by de Mazancourt and Dieckmann (2004) the space of possible carrying capacities ranges from 0 to 10. As mentioned above, no individual-based derivation of the logistic equation is known to us that can produce a weak trade-off in $\boldsymbol{K}$ for this choice of parameters. Since a weak trade-off is a prerequisite for evolutionary branching in their model, this seems, at least from the viewpoint of individual-level traits, contrary to the statement of the authors, a very unlikely outcome. We want to emphasize that our objection against the specific example does not detract from the eminent suitability of their methodology for analyzing situations where little knowledge is available on the mapping from individual-level traits to population-level parameters. An analysis along their lines produces graphical conditions that a trade-off curve has to fulfill for a specific outcome to be realized.

Ultimately the nature of variation at any given level is an empirical question and it might very well be that in a certain species a pattern of variation is found that matches the assumed variation in the above mentioned studies without that this pattern is derived from considerations at the level of the individual. Therefore, theoretical studies of an a priori chosen pattern of variation in population-level parameters without deriving it from an underlying level can still be useful. However, such a match would seem to be a lucky coincidence and should not form the basis of a research program. Instead, we suggest the following approach. Evolutionary predictions should be derived from models that assume evolutionary change at the level of individual-based traits. In order to get a broader picture one should get a collection of different individual-based derivations. In a second step one can classify the traits occurring in these derivations with respect to how variation at this level is mapped to the level of demographic traits and therefore result in the same class of evolutionary dynamics.

\section{The Evolution of Resource Specialization}

Finally we want to draw attention to the results of our evolutionary analysis as such. In the face of trade-offs, theory predicts two qualitatively different evolutionary outcomes. Natural selection can either lead towards an intermediate phenotype where the gain from improving one trait is exactly balanced by the loss through the accompanying change in another trait or to an extreme phenotype at the boundary of the trait space. The evolution of habitat specialization in terms of the size of the interaction neighborhood $a$ shows a marked difference in the continuous

and the discrete time model. In the continuous time model, where fast migration between habitats is assumed, the generalist is selected for in case of weak trade-offs, while specialists are selected 
for in case of strong trade-offs. This scenario coincides with the intuition of many evolutionary ecologists about the evolution of resource specialization (e.g., Benkman 1993; Robinson 2000) and with Levins' predictions for evolution in an environment stable in time but heterogeneous in space (Levins 1962). By contrast, in the discrete time model, where juvenile dispersal and no migration is assumed, specialists are also selected for in combination with weak trade-offs. This scenario shows that Levins' result does not hold generally but only when fitness is a linear function of the traits considered evolvable.

\section{Acknowledgments}

CR was supported by the Research Council for Earth and Life Sciences (ALW), which is subsidized by the Netherlands Organization for Scientific Research (NWO) and by a Discovery Grant to Peter Abrams from the Natural Sciences and Engineering Research Council of Canada. The authors thank Michel Durinx, Frans Jacobs and Tom Van Dooren for discussions and Peter Abrams for pointing out relevant literature. 


\section{REFERENCES}

Arnold, S. J. 1992. Constraints on phenotypic evolution. The American Naturalist 140:S85-S107.

Benkman, C. W. 1993. Adaptation to single resources and the evolution of crossbill (Loxia) diversity. Ecological Monographs 63:305-325.

Berryman, A. 1992. Intuition and the logistic equation. Trends in Ecology and Evolution 7:316.

Brännström, r. and Sumpter, D. 2005. The role of competition and clustering in population dynamics. Proceedings of the Royal Society London B 272:2065-2072.

Charlesworth, B. 1971. Selection in density-regulated populations. Ecology 52:469-474.

Charlesworth, B. 1990. Optimization models, quantitative genetics, and mutation. Evolution 44:520-538.

Charnov, E. L. 1989. Phenotypic evolution under fisher's fundamental theorem of natural selection. Heredity 62:113-116.

de Mazancourt, C. and Dieckmann, U. 2004. Trade-off geometries and frequency-dependent selection. The American Naturalist 164:765-778.

Diekmann, O. and Heesterbeek, J. A. P., 2000. Mathematical Epidemiology of Infectious Diseases: Model Building, Analysis and Interpretation. Wiley, Chichester.

Edelstein-Keshet, L., 1988. Mathematical Models in Biology. The Random House/Birkhäuser Mathematics Series.

Egas, M., Dieckmann, U., and Sabelis, M. W. 2004. Evolution restricts the coexistence of specialists and generalists - the role of trade-off structure. The American Naturalist 163:518-531.

Eshel, I. 1983. Evolutionary and continuous stability. Journal of Theoretical Biology 103:99-111.

Gatto, M. 1993. The evolutionary optimality of oscillatory and chaotic dynamics in simple population models. Theoretical Population Biology 43:310-336.

Geritz, S. A. H. and Kisdi, E. 2004. On the mechanistic underpinning of discrete-time population models with complex dynamics. Journal of Theoretical Biology 228:261-269.

Gurney, W. S. C. and Nisbet, R. M., 1998. Ecological Dynamics. Oxford University Press.

Gyllenberg, M. 2005. Book review: "differential equations and mathematical biology" by b. d. jones and d. s. sleeman. Mathematical Biosciences 193:19-24.

Hanski, I., 1999. Metapopulation Ecology. Oxford University Press, Oxford, U.K. 
Kooi, B. W., Boer, M. P., and Kooijman, S. A. L. M. 1998. On the use of the logistic equation in models of food chains. Bulletin of Mathematical Biology 60:231-246.

Kuno, E. 1991. Some strange properties of the logistic equation defined with $r$ and $k$ : Inherent defects or artifacts? Researches on Population Ecology 33:33-39.

Levins, R. 1962. Theory of fitness in a heterogeneous environment. i. the fitness set and the adaptive function. The American Naturalist 96:361-373.

MacArthur, R. and Wilson, E., 1967. Island Biogeography. Princeton University Press, Princeton, New Jersey.

MacArthur, R. H., 1972. Geographical Ecology. Harper \& Row, NY.

Matessi, C. and Gatto, M. 1984. Does k-selection imply prudent predation? Theoretical Population Biology 25:347-363.

May, R. M. and Oster, G. F. 1976. Bifurcation and dynamic complexity in simple ecological models. The American Naturalist 110:573-599.

Metz, J. A. J., Mylius, S. D., and Diekmann, O., 1996. When does evolution optimize? on the relation between types of density dependence and evolutionarily stable life history parameters. IIASA working paper WP-96-04, available at http://www.iiasa.ac.at/Research/ADN/Series.html.

Metz, J. A. J., Nisbet, R. M., and Geritz, S. A. H. 1992. How should we define 'fitness' for general ecological scenarios? Trends in Ecology and Evolution 7:198-202.

Mylius, S. D. and Diekmann, O. 1995. On evolutionary stable life histories, optimization and the need to be specific about density dependence. Oikos 74:218-224.

Olson, M. 1992. Intuition and the logistic equation. Trends in Ecology and Evolution 7:314.

Parvinen, K. and Egas, M. 2004. Dispersal and the evolution of specialization in a two-habitat type metapopulation. Theoretical Population Biology 66:233-248.

Ricker, W. E. 1952. Stock and recruitment. Journal of the Fisheries Research Board of Canada 11:559-623.

Robinson, B. W. 2000. Trade offs in habitat-specific foraging efficiency and the nascent adaptive divergence of sticklebacks in lakes. Behaviour 137:865-888.

Roughgarden, J. 1971. Density-dependent natural selection. Ecology 52:453-468.

Royama, T., 1992. Analytical Population Dynamics. Number 10 in Population and Community Biology Series. Chapman \& Hall. 
Rueffler, C., Van Dooren, T. J. M., and Metz, J. A. J. 2004. Adaptive walks on changing landscapes: Levins' approached extended. Theoretical Population Biology 65:165-178.

Schoener, T. W. 1973. Population growth regulated by intraspecific competition for energy and time: Some simple representations. Theoretical Population Biology 4:56-84.

Sumpter, D. J. T. and Broomhead, D. S. 2001. Relating individual behaviour to population dynamics. Proceedings of the Royal Society London, B 268:925-932.

Thieme, H., 2003. Mathematics in Population Biology. Princeton University Press, Princeton, New Jersey.

Wilson, D. S. and Yoshimura, J. 1994. On the coexistence of specialists and generalists. The American Naturalist 144:692-707. 


\section{Appendix A: Fast Migration Between Habitats}

The population dynamics of one consumer species exploiting two different habitats can be described by the following system of coupled differential equations:

$$
\begin{aligned}
& \frac{\mathrm{d} N_{1}}{\mathrm{~d} t}=m_{12} N_{2} \frac{A_{2}}{A_{1}}-m_{21} N_{1}+b_{1} N_{1}-d_{1} N_{1} \\
& \frac{\mathrm{d} N_{2}}{\mathrm{~d} t}=m_{21} N_{1} \frac{A_{1}}{A_{2}}-m_{12} N_{2}+b_{2} N_{2}-d_{2} N_{2} .
\end{aligned}
$$

$N_{1}$ and $N_{2}$ denote the population density in habitat one and two, respectively. Individuals migrate at rate $m_{i j}$ from habitat $j$ to habitat $i$. Absolute habitat size is denoted by $A_{i}$. In each habitat individuals reproduce and die at the habitat-specific rates $b_{i}$ and $d_{i}$, respectively. If we assume that migration rates are high in comparison to the birth and death rates, we can calculate the equilibrium distribution of the population over the two habitats as $\hat{N}_{1}=\hat{N}_{2} A_{2} m_{12} / A_{1} m_{21}$. Combining this with $n=A_{1} N_{1}+A_{2} N_{2}$, where $n$ denotes the total population size, we find that $p_{1}=A_{1} \hat{N}_{1} / \hat{n}=$ $m_{12} /\left(m_{21}+m_{12}\right)$ and $p_{2}=A_{2} \hat{N}_{2} / \hat{n}=m_{21} /\left(m_{21}+m_{12}\right)$.

In order to write the model purely in terms of individual-level traits, we assume that the habitat-specific death rates increase linearly with the number of competitors and that therefore the realized rate of increase of a consumer with $n$ competitors in its competition neighborhood of size $a$ is given by $r_{n}=b_{0}-\left(d_{0}+l n\right)$. Here $l$ is a positive constant. It describes the sensitivity

to competition such that the sensitivity is increasing with increasing values of $l$. If we substitute $r_{0}$ for $b_{0}-d_{0}$ we can rewrite the realized rate of increase as $r_{n}=r_{0}-l n$. The expected rate of increase is then given by

$$
E[r]=r_{0}-l \sum_{n} n P(n)=r_{0}-l \bar{n}=r_{0}-l N a=r_{0}\left(1-\frac{l a N}{r_{0}}\right),
$$

i.e., $K=r_{0} / l a$.

\section{Appendix B: Mapping of the Trade-Off}

Consider a trade-off between $a_{1}$ and $a_{2}$ that can be described by the function $a_{2}\left(a_{1}\right)$. This trade-off curve is translated into a curve $K_{2}\left(K_{1}\right)$ by the map $K_{2}\left(a_{2}\left(a_{1}\left(K_{1}\right)\right)\right)$. This map has to be derived from equation (12) and (10) or (11). To study the curvature of the trade-off in $\boldsymbol{K}$, we have to differentiate $K_{2}\left(a_{2}\left(a_{1}\left(K_{1}\right)\right)\right)$ twice:

$$
\frac{\mathrm{d}^{2} K_{2}\left(a_{2}\left(a_{1}\left(K_{1}\right)\right)\right)}{\mathrm{d} K_{1}^{2}} \propto 2 \frac{\mathrm{d} a_{2}}{\mathrm{~d} a_{1}}\left(\frac{\mathrm{d} a_{2}}{\mathrm{~d} a_{1}} \frac{1}{a_{2}}-\frac{1}{a_{1}}\right)-\frac{\mathrm{d}^{2} a_{2}}{\mathrm{~d} a_{1}^{2}} .
$$

This gives us an expression for the shape of the trade-off in terms of the first two derivatives of $a_{2}\left(a_{1}\right)$. Further analytical results can be obtained for specific parameterizations of the trade-off 
curve. Here we choose equation (12) which can also be written as

$$
1=\left(\frac{a_{1 \max }-a_{1}}{a_{1 \mathrm{var}}}\right)^{z}+\left(\frac{a_{2 \max }-a_{2}}{a_{2 \mathrm{var}}}\right)^{z} .
$$

We can derive the trade-off function $a_{2}\left(a_{1}\right)$ by solving equation (B2) for $a_{2}$. The first two derivatives of this function can be simplified using equation (12) and equation(B2) to:

$$
\frac{\mathrm{d} a_{2}}{\mathrm{~d} a_{1}}=-\frac{(1-\theta) \theta^{\frac{1}{z}} a_{2 \mathrm{var}}}{(1-\theta)^{\frac{1}{z}} \theta a_{1 \mathrm{var}}} \quad \text { and } \quad \frac{\mathrm{d}^{2} a_{2}}{\mathrm{~d} a_{1}^{2}}=\frac{(1-\theta) \theta^{\frac{1}{z}} a_{2 \mathrm{var}}(z-1)}{(1-\theta)^{\frac{2}{z}} \theta^{2} a_{1 \mathrm{var}}^{2}} .
$$

Now we are able to simplify condition (B1) using equation (B3):

$$
\frac{\mathrm{d}^{2} K_{2}\left(a_{2}\left(a_{1}\left(K_{1}\right)\right)\right)}{\mathrm{d} K_{1}^{2}} \propto 1-z+2\left[\theta \frac{a_{1 \mathrm{var}}(1-\theta)^{\frac{1}{z}}}{a_{1 \max }-a_{1 \operatorname{var}}(1-\theta)^{\frac{1}{z}}}+(1-\theta) \frac{a_{2 \operatorname{var}} \theta^{\frac{1}{z}}}{a_{2 \max }-a_{2 \operatorname{var}} \theta^{\frac{1}{z}}}\right]
$$

This condition depends on the magnitude of $z$ relative to 1 plus two times some complicated expression in brackets. If both $a_{i \text { var }}$ are small, then the term in brackets is small as well, and the sign of the second derivative will be determined by the difference between 1 and $z$. In this case, whether $K_{2}\left(a_{2}\left(a_{1}\left(K_{1}\right)\right)\right)$ is a strong or a weak trade-off is inherited for most values of $z$ from the corresponding property in the underlying trade-off $a_{2}\left(a_{1}\right)$. By contrast, if both $a_{i v a r}$ are large, i.e., very similar to $a_{i \max }$, then the term in brackets will be large as well and can dominate the whole expression. In this case moderately weak trade-offs in $\boldsymbol{a}$ will be mapped into strong trade-offs in $\boldsymbol{K}$. When $a_{i \text { var }}$ approaches infinity, that is, when $K_{i \text { imin }}$ approaches zero, then $K_{2}\left(K_{1}\right)$ will be a convex trade-off for any value of $z$.

\section{Appendix C: Analytical Results for Evolutionary Analysis}

First we present some analytical results for the case of fast migration and mutational change in $\boldsymbol{K}$. These derivations are very similar to those in appendix B and will not be repeated in as much detail. The optimization criterion is given by $\hat{N}$ (eq. 4). Differentiating $\hat{N}$ with respect to $K_{1}$ (where $K_{2}$ is considered a function of $K_{1}$ ) reveals that critical points of $\hat{N}$ are given by $\mathrm{d} K_{2} / \mathrm{d} K_{1}=-\left(p_{1}^{2} r_{1} K_{2}^{2}\right) /\left(p_{2}^{2} r_{2} K_{1}^{2}\right)$. To see whether these critical points are maxima or minima of $\hat{N}$ we need to differentiate $\hat{N}$ twice. It appears that we get a more tractable result if we differentiate $\hat{N}^{\prime}:=-1 / \hat{N}$, an expression that has minima and maxima for the same values as $\hat{N}$. Differentiating $\hat{N}^{\prime}$ twice with respect yields

$$
\frac{\mathrm{d}^{2} \hat{N}^{\prime}}{\mathrm{d} K_{1}^{2}}=\frac{p_{2}^{2} r_{2}}{K_{2}^{2}} \frac{\mathrm{d}^{2} K_{2}}{\mathrm{~d} K_{1}^{2}}-\frac{2 p_{1}^{2} r_{1}}{K_{1}^{3}}-\frac{2 p_{2}^{2} r_{2}}{K_{2}^{3}}\left(\frac{\mathrm{d} K_{2}}{\mathrm{~d} K_{1}}\right)^{2}
$$

When we evaluate this derivative a the critical points of $\hat{N}$ we can replace the middle term with $-\left(\mathrm{d} K_{2} / \mathrm{d} K_{1}\right) 2 r_{2} p_{2}^{2} /\left(K_{2}^{2} K_{1}\right)$. This allows us factor out $p_{2} r_{2} / K_{2}^{2}$ from the right hand side of equation 
(C1). After replacing $\mathrm{d} K_{2} / \mathrm{d} K_{1}$ and $\mathrm{d}^{2} K_{2} / \mathrm{d} K_{1}^{2}$ with their explicit expressions, which can be derived analogous to equation (B3), we get

$$
\frac{\mathrm{d}^{2} \hat{N}^{\prime}}{\mathrm{d} K_{1}^{2}} \propto 1-z-2\left(\theta \frac{K_{1 \mathrm{var}}(1-\theta)^{\frac{1}{z}}}{K_{1 \min }+K_{1 \mathrm{var}}(1-\theta)^{\frac{1}{z}}}+(1-\theta) \frac{K_{2 \operatorname{var}} \theta^{\frac{1}{z}}}{K_{2 \min }+K_{2 \operatorname{var}} \theta^{\frac{1}{z}}}\right) .
$$

For $z>1$ (weak trade-offs) this expression is always negative and therefore critical points are maxima. For $z<1$ the sign of expression (C2) depends on the term in brackets. When this term is larger than 0.5 , then $\mathrm{d}^{2} \hat{N}^{\prime} / \mathrm{d} K_{1}^{2}$ will be negative for any value of $z$ and the critical point will be again a maximum. This is the case when the $K_{i \text { min }}$ 's are sufficiently small. In case of symmetric parameter values for $r, p, K_{\min }, K_{\max }$, we find a critical point of the optimization criterion at $\theta=0.5$. A sufficient condition for $\mathrm{d}^{2} \hat{N}^{\prime} / \mathrm{d} K_{1}^{2}$ to be negative is then given by $K_{\min }<K_{\operatorname{var}} 0.5^{\frac{1}{z}}$. However, when both $K_{i \text { var }}$ are sufficiently small, then the term in brackets will be close to zero and the difference between one and $z$ dominates the term in brackets. The optimization criterion has then the same qualitative curvature as the trade-off $K_{2}\left(K_{1}\right)$. In the limit $K_{i \mathrm{var}}=0$, the term in brackets becomes zero and a strong trade-off in $\boldsymbol{K}$ corresponds to a minimum in $\hat{N}$ and a weak trade-off in $\boldsymbol{K}$ corresponds to a maximum in $\hat{N}$.

For the model with juvenile dispersal and symmetric parameter values $\left(p_{1}=1 / 2=p_{2}, r_{1}=\right.$ $\left.r=r_{2}, K_{1 \min }=K_{2 \min }, K_{1 \mathrm{var}}=K_{2 \mathrm{var}}, a_{1 \max }=a_{2 \max }, a_{1 \mathrm{var}}=a_{2 \mathrm{var}}, R_{01}=R_{02}, k_{1}=k=k_{2}\right)$ we can prove that the bifurcation from a repelling generalist to a generalist that is a CSS occurs for $z<1$ when the trade-off is directly in $\boldsymbol{K}$, and for $z>1$ when the trade-off is in $\boldsymbol{a}$. Under the assumption of symmetry the first derivative of the fitness function (eq. [16] and eq. [17]) equals zero at the generalists trait where $K_{1}=K=K_{2}$ and $a_{1}=a=a_{2}$, respectively. At these trait values $\mathrm{d} K_{2} / \mathrm{d} K_{1}=-1$ and $\hat{N}=2 K$ in case of a trade-off directly in $\boldsymbol{K}$ and $\mathrm{d} a_{2} / \mathrm{d} a_{1}=-1$ and $\hat{N}=2 r /(a(1-k))$ in case of a trade-off in $\boldsymbol{a}$. The bifurcation point is given by the $z$-value where the second derivative of the fitness function (eq. [16] and eq. [17]) equals zero: $\mathrm{d}^{2} w\left(\boldsymbol{K}^{\prime}, \boldsymbol{K}\right) / \mathrm{d} K_{1}^{\prime 2}=0$ and $\mathrm{d}^{2} w\left(\boldsymbol{a}^{\prime}, \boldsymbol{a}\right) / \mathrm{d} a_{1}^{\prime 2}=0$, respectively. Under the above mentioned conditions we can derive that the bifurcation points are characterized by

$$
\mathrm{d}^{2} K_{2} / \mathrm{d} K_{1}^{2}=2(2-r) / K
$$

and

$$
\mathrm{d}^{2} a_{2} / \mathrm{d} a_{1}^{2}=2 r / a,
$$

respectively. The right hand side of eq. (C3) is positive for $r<2$. Hence, for stable population dynamical equilibria the bifurcation occurs for a convex trade-off in $\boldsymbol{K}$. As explained in the section on the mapping of trade-offs, this corresponds to a strong trade-off in $\boldsymbol{K}(z<1)$. The right hand side of eq. (C4) is also a positive number, thus, indicating again that the bifurcation occurs for a convex trade-off. However, for this case where the trade-off is assumed in $\boldsymbol{a}$, a convex trade-off corresponds to a weak trade-off $(z>1)$. 


\section{Figure Legends}

Fig. 1.- Distribution of phenotypes in the two-dimensional $\left(\alpha_{1}, \alpha_{2}\right)$-trait space. Solid lines correspond to a constraint or trade-off beyond which no viable phenotypes can occur. Since fitness is decreasing in both $\alpha_{1}$ and $\alpha_{2}$ the set of possible phenotypes lies above and to the right of the constraint. The right panel shows the location of the phenotypes and the constraint after applying the mapping $\left(\alpha_{1}, \alpha_{2}\right) \mapsto\left(\left(b_{1}-d_{1}\right) / \alpha_{1},\left(b_{2}-d_{2}\right) / \alpha_{2}\right)=\left(K_{1}, K_{2}\right)$. Because fitness is increasing in both $K_{1}$ and $K_{2}$ the distribution of phenotypes lie below and to the left of the constraint. Note that the trade-off has changed from weak to strong (see text for further explanation). In the $\left(\alpha_{1}, \alpha_{2}\right)$-space the phenotypes have relative low values for both habitats, indicating a high degree of adaptation, while in the $\left(K_{1}, K_{2}\right)$-space the phenotypes again correspond to low values, now indicating a low degree of adaptation. Parameter values are chosen such that $b_{i}-d_{i}=1$.

Fig. 2.- Mapping of five different trade-off curves for the habitat-specific size of competition neighborhoods $a_{i}$ onto trade-off in carrying capacities $K_{i}$ through function given by equation (10) ( $\mathrm{a} \& \mathrm{~b}$ ), and vice versa through the inverse function (c \& d). The left graph in each pair shows tradeoffs for five values of the curvature parameter $z$ before the application of the mapping while the right graph shows trade-offs as a result of the mapping. Curves with the same gray scale correspond to the same value of $z \in\{4,2,1,0.5,0.25\}$ with $z$ decreasing with lighter coloration. Other parameters: $\boldsymbol{b}=(0.3,0.3), \boldsymbol{d}=(0.1,0.1), \boldsymbol{p}=(0.5,0.5)$, (a) $\boldsymbol{a}_{\max }=(0.2,0.2), \boldsymbol{a}_{\mathrm{var}}=(0.1,0.1), \boldsymbol{l}=(10,10)$, (b) $\boldsymbol{a}_{\max }=(1,1), \boldsymbol{a}_{\mathrm{var}}=(0.9,0.9), \boldsymbol{l}=(2,2),(\mathrm{c}) \boldsymbol{K}_{\min }=(0.1,0.1), \boldsymbol{K}_{\mathrm{var}}=(0.1,0.1), \boldsymbol{l}=(10,10),(\mathrm{d})$ $\boldsymbol{K}_{\min }=(0.1,0.1), \boldsymbol{K}_{\mathrm{var}}=(0.9,0.9), \boldsymbol{l}=(2,2)$.

Fig. 3.- Bifurcation diagram of extrema of optimization criterion as a function of curvature parameter $z$ for model with: (a \& b) fast migration between habitats (logistic equation), (c \& d) juvenile dispersal (Ricker equation). Ordinate shows value of the specialization coefficient $\theta$. (a \& c) Trade-off directly in $\boldsymbol{K}$. (b \& d) Trade-off in $\boldsymbol{a}$. Solid lines indicate maxima of the optimization criterion and correspond to CSSs while dashed lines indicate minima of the optimization criterion and correspond to evolutionary repellors. The gray vertical line in (b) indicates that for $\theta=1$ all trait combination are selectively neutral. Arrows indicate the direction of evolutionary change. Parameter values: $\boldsymbol{p}=(0.5,0.5),(\mathrm{a} \& \mathrm{c}) \boldsymbol{K}_{\min }=(0.1,0.1), \boldsymbol{K}_{\mathrm{var}}=(0.4,0.4), \boldsymbol{r}=(0.2,0.2),(\mathrm{b} \&$ d) $\boldsymbol{a}_{\max }=(0.5,0.5), \boldsymbol{a}_{\mathrm{var}}=(0.4,0.4), \boldsymbol{l}=(4,4)$, (b) $\boldsymbol{l}=(4,4), \boldsymbol{b}=(0.3,0.3), \boldsymbol{d}=(0.1,0.1)$, (d) $\boldsymbol{k}=(0.1,0.1), \log \boldsymbol{R}_{0}=(0.2,0.2)$. 
Fig. 4.- Fitness contour plots for model with: (a-c) fast migration between habitats (logistic equation) and (d-f) juvenile dispersal (Ricker equation). Fitness contours represent values of optimization criteria as function of $K_{1}$ and $K_{2}$ (a, c, d, f) or $a_{1}$ and $a_{2}$ (b, e). Lighter coloration indicates higher values of the optimization criterion and therefore higher fitness. Trade-offs with different values of the curvature parameter $z$ are plotted on top of the fitness landscape. For a given trade-off curve the optimal phenotype is given by the point on the trade-off curve lying on the highest contour. The color of the circles at the position of the habitat generalist indicates evolutionary properties: black circle $=\mathrm{CSS}$, white circle $=$ repellor, gray circle $=$ selectively neutral with respect to all other traits on the trade-off. In (a \& d) the trade-off is assumed directly in $\boldsymbol{K}$. In (b \& e) the trade-off is assumed in $\boldsymbol{a}$. In (c \& f) the trade-off in $\boldsymbol{K}$ is derived from the underlying trade-off in $\boldsymbol{a}$. These latter two representations necessarily give the same result. Parameter values as in figure 3. 

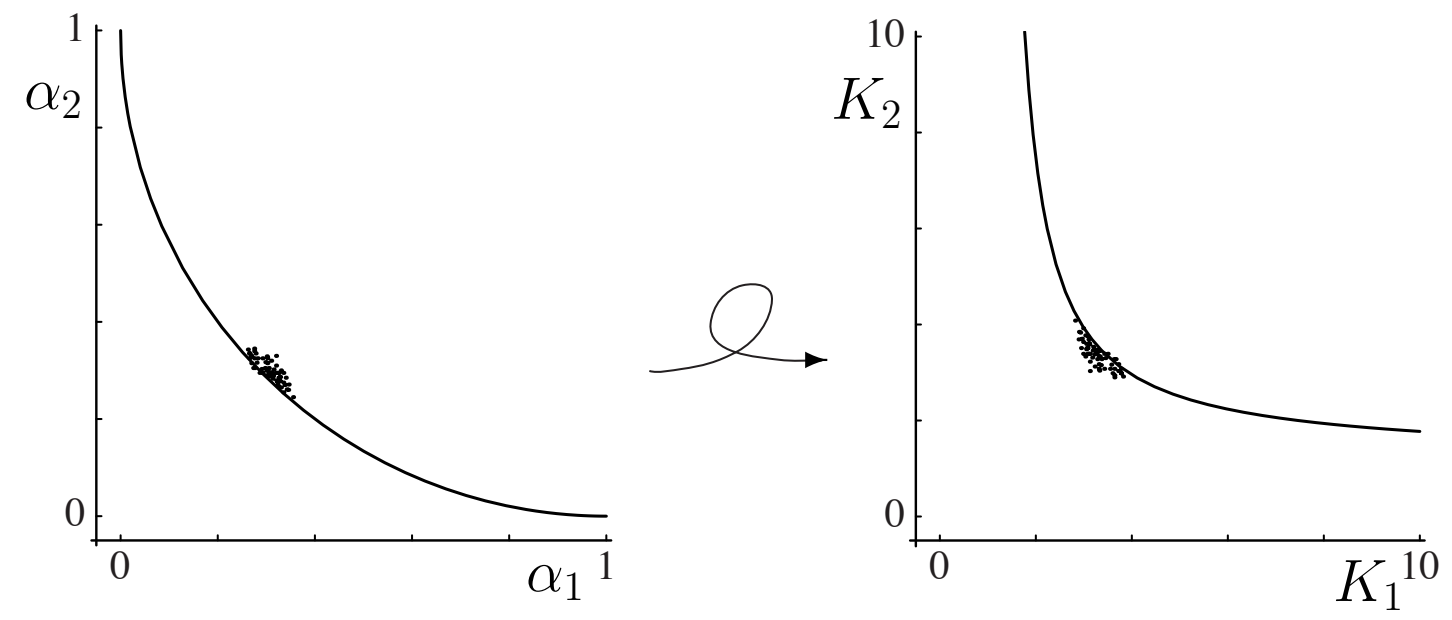

Figure 1 


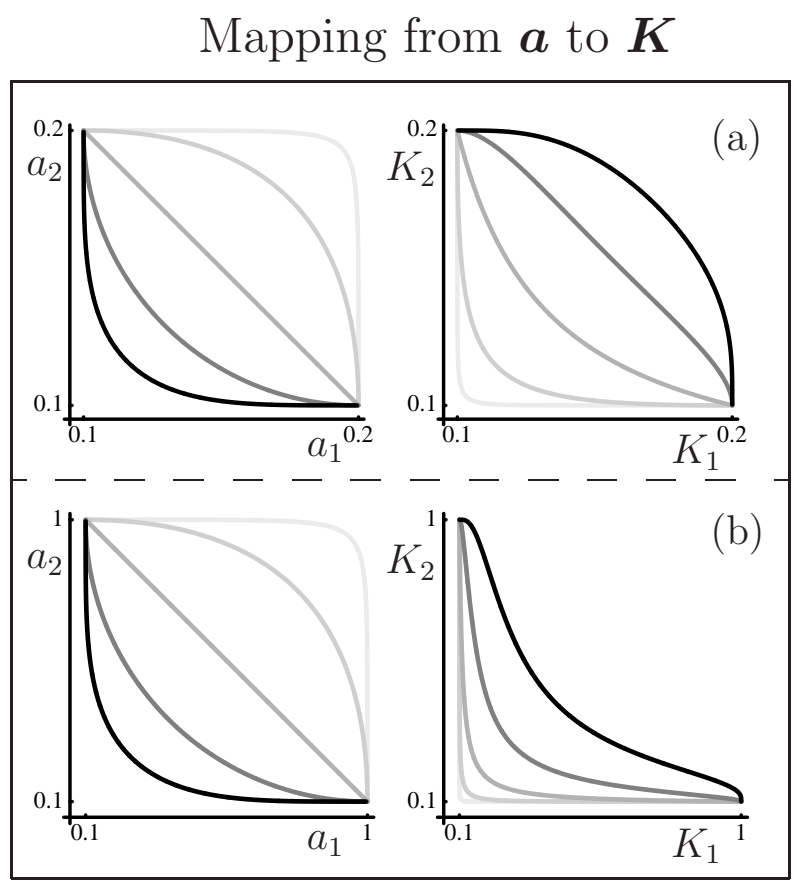

Mapping from $\boldsymbol{K}$ to $\boldsymbol{a}$

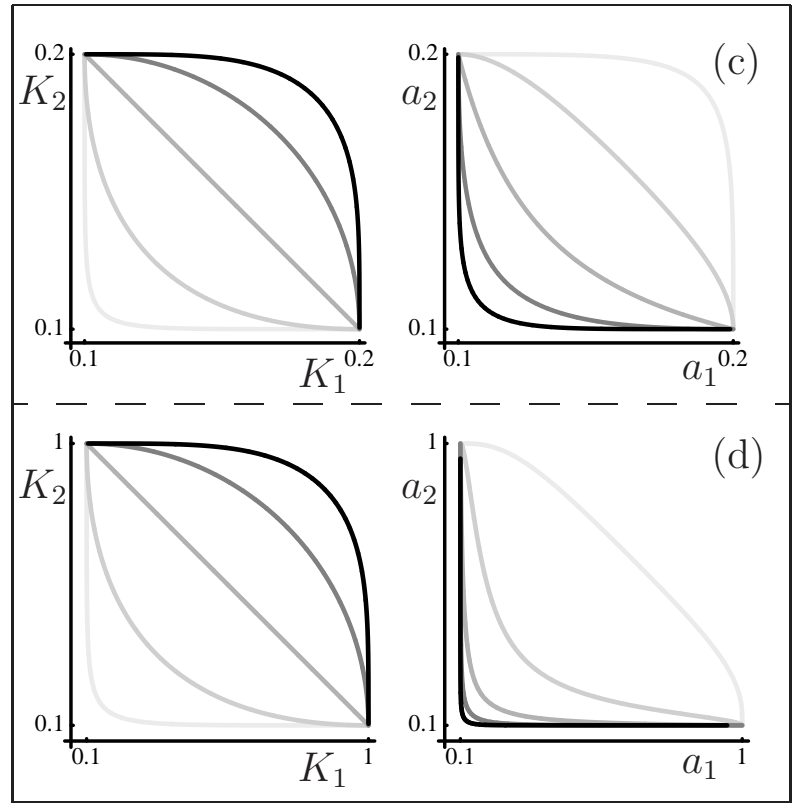

Figure 2 
Trade-Off in $\boldsymbol{K}$
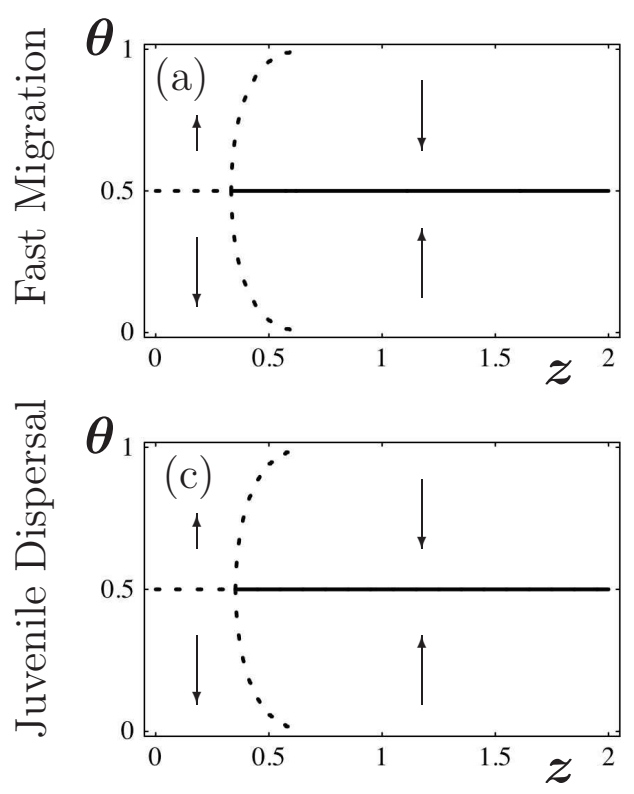

Trade-Off in $\boldsymbol{a}$
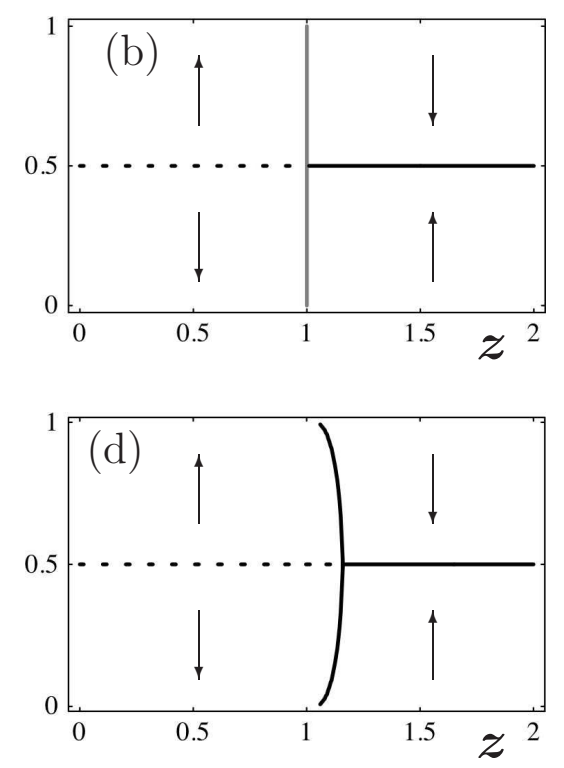

Figure 3 
(a)

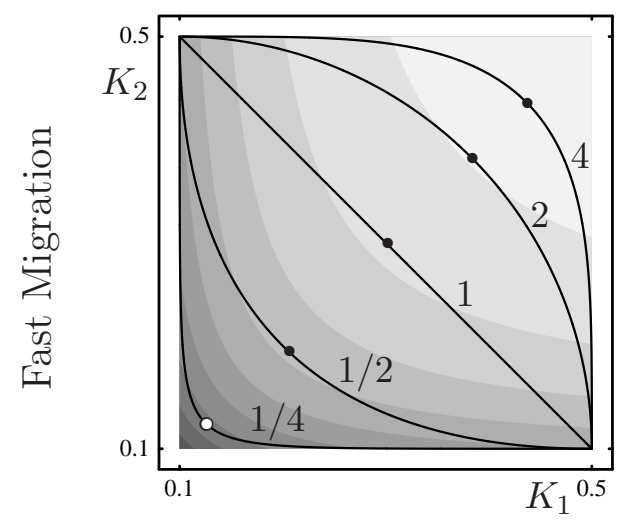

(d)

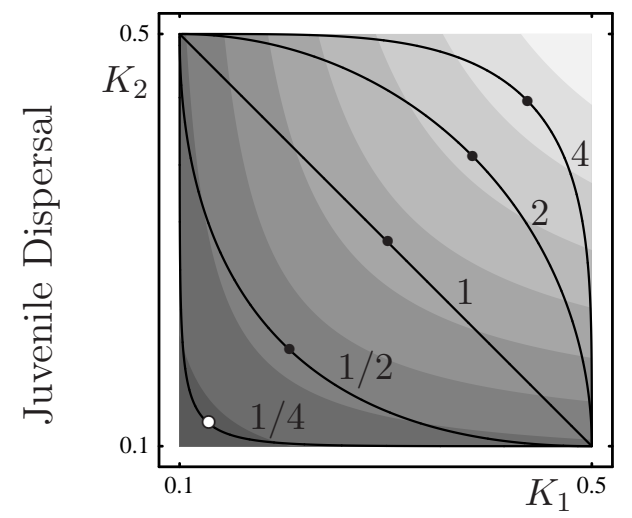

(b)

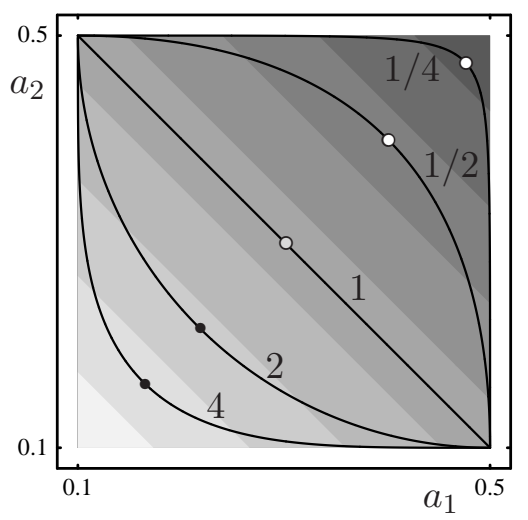

(e)

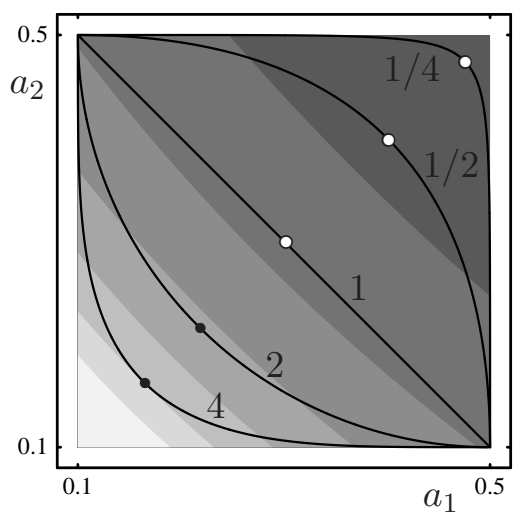

(c)

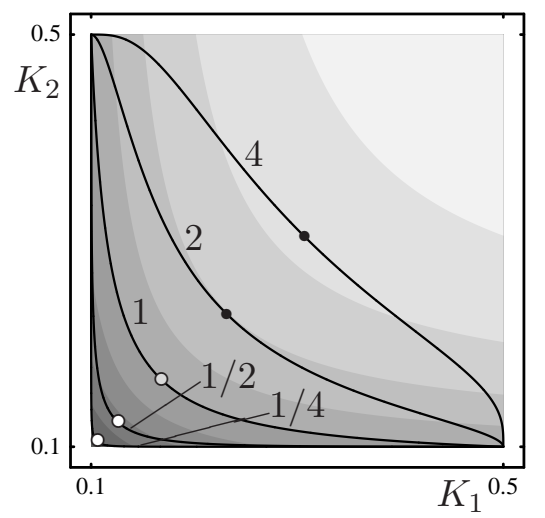

(f)

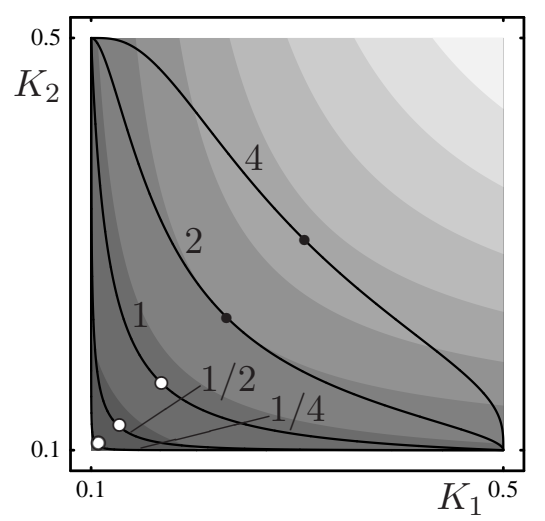

